\title{
Digitalkompetenz und Data Literacy als professionelle Kompetenzen für Soziale Arbeit im Zeitalter des digitalen Kapitalismus: Der Einfluss der Digitalisierung auf Lehre und Ausbildungsprofile in der Sozialen Arbeit
}

Jahrestagung der Deutschen Gesellschaft für Soziale Arbeit (DGSA), 26.-27. April, Stuttgart

Joachim K. Rennstich*

26 April 2019

\section{Zusammenfassung}

Die steigenden Anforderungen der Evidenz-basierten Ausgestaltung in professionellen Kontexten von Sozialer Arbeit stellt zunehmende Herausforderungen an die Ausbildungsprofile in der Lehre dar. Der zunehmende Trend zu quantitativen Formen der Vermittlung von Evidenz wird durch die Digitalisierung noch weiter beschleunigt und manifestiert die Notwendigkeit der Verbindung qualitativer und quantitativer Kompetenzen zur Ausbildung einer umfassenden Data Literacy, also dem verständigen Umgang mit Daten und deren Interpretation. Gerade in der Sozialen Arbeit ist es jedoch wichtig, Datenermittlung und -interpretation als umfassendes Konzept zu vermitteln. Am Beispiel der sozialraumorientierten Arbeit und der Methode der Netzwerkanakyse stellt dieser Beitrag neue Möglichkeiten einer Verbindung von qualitativen und quantitativen Methoden in der Netzwerkanalyse vor und diskutiert notwendige Kompetenzprofile sowie Voraussetzungen für eine erfolgreiche Kompetenzvermittlung in der Lehre unter Berücksichtigung methodischer Kenntnisse, ethischer Fragestellungen und kritischer Reflexionsfähigkeit in einer von Digitalisierung geprägten sozialen Wirklichkeit und vom digitalen Kapitalismus geprägten gesellschaftlichen Gesamtkontexts.

\section{Notwendige Kompetenzerweiterung oder Zeitgeist Hype? Neue Herausforderungen für professionelles Handeln in der Sozialen Arbeit}

Die steigenden Anforderungen der evidenzbasierten Ausgestaltung in professionellen Kontexten von Sozialer Arbeit stellt zunehmende Herausforderungen an die Ausbildungsprofile in der

*CVJM-Hochschule (YMCA University of Applied Sciences), Hugo-Preuß-Str. 40, 34131 Kassel, GERMANY. E-Mail: joachim@rennstich.com, Web: www.rennstich.de 
Lehre dar. Dies gilt gleichermaßen für die Handlungskompetenzvermittlung in der Ausbildung in Bachelor und Master Studiengängen, sowie die Ausbildung des akademischen Nachwuchses bezogen auf Forschungs- und Lehrvermittlungskompetenz. Dieser zunehmende Trend zu quantitativen Formen der Vermittlung von Evidenz wird durch die Digitalisierung noch weiter beschleunigt und manifestiert die Notwendigkeit der Verbindung qualitativer und quantitativer Kompetenzen zur Ausbildung einer umfassenden „Data Literacy“, also dem verständigen Umgang mit Daten und deren Interpretation. Die zunehmend fortschreitende Digitalisierung unseres sozialen Alltags erfordert jedoch zusätzlich auch einen dezidierten Fokus auf die Erweiterung des Kompetenzprofils von Akteur*innen der Sozialen Arbeit bezüglich ihrer Sprachund Vermittlungsfähigkeit in gänzlich digitalisierten oder hybriden Kommunikationskontexten, also der Vermittlung einer „Digital Literacy“. Dieser Text geht daher zunächst auf die durch den Wandel hin zu einem digitalen Kapitalismus hervorgerufenen Transformation von sozialem Raum und Kernelementen sozialer Interaktion innerhalb dieses Raumes ein und beschreibt diese Anhand der soziologischen Konzepte von Sozialkapital, Vertrauen und Agency.

Gerade in der Sozialen Arbeit ist es jedoch wichtig, Datenermittlung und -interpretation als umfassenden Konzept zu vermitteln. Während in der Vergangenheit das Hauptaugenmerk hauptsächlich auf qualitative Formen der Datenerhebung und -analyse gelegt wurde, werden zunehmend von Akteuren der Sozialen Arbeit Kompetenzen verlangt, die eine sinnvolle Verbindung dieser qualitativen Methoden mit quantitativen Methoden in einer zunehmend durch die Digitalisierung vieler Prozesse geprägten Arbeits- und Forschungswelt ermöglichen. Am Beispiel der sozialraumorientierten Arbeit stellt dieser Beitrag neue Möglichkeiten einer Verbindung von qualitativen und quantitativen Methoden in der Netzwerkanalyse vor und diskutiert notwendige Kompetenzprofile sowie Voraussetzungen für eine erfolgreiche Kompetenzvermittlung in der Lehre unter Berücksichtigung methodischer Kenntnisse, ethischer Fragestellungen und kritischer Reflexionsfähigkeit in einer von Digitalisierung geprägten sozialen Wirklichkeit und vom digitalen Kapitalismus geprägten gesellschaftlichen Gesamtkontexts.

\section{Soziologische Transformation: Herausforderung Digital- isierung am Beispiel des Sozialraumansatzes}

Soziologische Grundlagentheorien sind von großer Bedeutung für die erfolgreiche Soziale Arbeit in der Praxis vor allem hinsichtlich der Kontexte — also „Struktur“ —, in denen sich Akteure der Sozialen Arbeit bewegen, aber auch hinsichtlich der Akteure — den „Subjekten“ im soziologischen Duktus - selber. Beide Ebenen, die Makroebene der Gesellschaft, genau so wie die Ebene des Individuums spielen eine wichtige Rolle, denn sie beeinflussen sich gegenseitig. Das Umfeld bestimmt nicht alleine die Handlungen von Menschen. Der Mensch ist aber in weiten Teilen von seinem oder ihrem Umfeld eben doch abhängig in seinen oder ihren Handlungsoptionen und dem, was wir heute als „Agency“ bezeichnen, also einem erweiterten Verständnis dessen, was individuelles und auch institutionelles Handeln beinhaltet und kennzeichnet (Graßhoff, 2013).

Vor allem im angelsächsischen Raum versucht die Theory der Agency diesen Dualismus, welcher Handlungen als sozialdeterminiert, Individuen als autonome Subjekte konzipiert, zwischen Struktur- und Subjektansatz zu überwinden (vgl. Giddens, 1979; Emirbayer \& Mische, 1998). In Europa hingegen sind wir mehr vertraut mit dem „Habitus“ Ansatz Bourdieus (Bour- 
dieu, 1974/2000, 19972017).

Im folgenden möchte ich besonders auf drei Aspekte dieser grundsätzlichen Problematik des „micro-macro-links“ näher eingehen - den Sozialraumansatz, das Konzept des Sozialkapitals und die Methode der Netzwerkanalyse — da sie alleinstehend aber auch gerade in ihrer Verbindung die Notwendigkeit soziologischen Wissens als Grundvoraussetzung für gute sozialarbeiterisches Handeln und die Praxis deutlich macht und gleichzeitig die Möglichkeiten in den methodischen Erweiterungen der hier behandelten Ansätze für eine Soziale Arbeit aufzeigt, die den sozialen Veränderungen moderner Gesellschaften des 21. Jahrhundert methodisch gut gewappnet ist.

Hierzu werden ich zunächst in kurzen Anrissen die Theorien des Sozialraum- und Sozialkapitalansatzes beleuchten, die durch die Globalisierung und Digitalisierung ausgelösten transformatorischen Umwälzungen unserer sozialen Realität thematisieren und im Anschluss näher auf die Methode der Netzwerkanalyse einzugehen und anhand kurzer praktischer Beispiele darstellen, wie diese Methode sowohl in Forschung als in der sozialarbeiterischen Praxis Anwendung finden kann.

Ausgehend von der Arbeit von Böhnisch \& Münchmeier (1990) stellt das Konzept der Sozialraumorientierung mittlerweile einen eigenständigen paradigmatischen Theorie- und Handlungsansatz in der Sozialen Arbeit dar. Im Wesentlichen gibt es hier zwei Herangehensweisen: (1) der Sozialraum als ein Aneignungsfeld vom Individuum ausgehend (Deinet, 2009b; Deinet \& Krisch, 2002) und (2) als praktische Entwicklungsaufgabe „vor Ort“ ausgehend vom Gemeinwesen als Umfeld (Budde u. a., 2006; Spatscheck, Arnegger, Kraus, \& Mattner, 2008).

Sozialraumorientierung kombiniert im Wesentlichen folgende bestehenden Konzepte der Sozialen Arbeit:

- Gemeinwesenarbeit,

- Empowerment,

- die Theorie des Sozialen Kapitals,

- das Konzept der lernenden Organisation,

- Aspekte der Neuen Steuerung und

- die Theorie der Lebensweltorientierung.

Somit erweitert die Sozialraumorientierung nicht nur die einzelnen bestehende Ansätze in ihrem Anwendungsbereich, sondern schafft durch deren Verbindung ein methodisch neues und erkennbar differenziertes Konstrukt.

Im Kontext des Sozialraumansatzes wird „Raum“ als das Ergebnis sozialen und organisatorischen Handelns begriffen. Dieser Raum rahmt das Handeln der Akteure massgeblich ein (Budde \& Früchtel, 2006, S. 27-28) und zwar in Form von:

- Interaktions- und Machtstrukturen,

- institutionalisierte normative Regulationssysteme, und

- Symbolsysteme.

Der Raum in diesem Sinne manifestiert sich zwar durchaus in seiner physischen Form; physische Orte nehmen beispielsweise einen zentralen Stellenwert für die Formierung von Strukturen ein etwa durch ihre limitierenden oder aktivierenden Aspekte des physischen Raums, 
man denke hier an die Begegnungen mit Peers oder eben auch der Bedeutung von „NichtBegegnungen“. Für das Gestaltwerden von Raum aus Sicht des Sozialraumansatzes sind andere Faktoren, die über den rein physischen Ort hinausgehen aber mindestens genauso bedeutsam, geht es doch um die Konstruiertet des Raums (vgl. Früchtel, Cyprian, \& Budde, 2013), um die Lebenswelt im Habermasschem Sinne, nämlich das Konglomerat aus sozialer Herkunft, Gruppenzugehörigkeiten und Umfeld (vgl. Habermas, 1981/1988, S. 182ff).

Der Ort als physische Lokalität und als Konstrukt stellt somit als Lebensraum und Lebenswelt ein zentrales Element für die Praxis der Sozialen Arbeit dar. Dieser Ort erfährt in der „Infosphäre" (infosphere) $)^{1}$ heute seine Erweiterung in die digitalen — also informationalisierten Orte, sowohl sozialer Interaktionen zwischen Menschen, als auch zwischen Personen und technologisch medialisierter Realität. Die Infosphäre stellt somit eine wesentlich Brücke zwischen physischen und digitalisierten Orten dar, die beide reale Lebenswelten von Personen, bewusst oder unbewusst, in immer stärkerem Maße darstellen (Floridi, 2014; vgl. auch Hammel, 2015; Wellman \& Haythornthwaite, 2002). Im folgenden Abschnitt will ich diesen Gedanken kurz am Beispiel der Offenen Kinder- und Jugendarbeit darstellen.

Die Sozialraumorientierung nimmt im Bereich der Offenen Kinder- und Jugendarbeit eine immer wesentlichere Rolle ein, u.a. mit dem Ansatz einer „sozialräumlichen Jugendarbeit“ (vgl. Deinet, 2009a; Krisch, 2009). Dieser Ansatz versteht sich durch die Anwendung vielfältiger partizipativer Methoden einer qualitativen Lebensweltanalyse auch als Form einer in der Jugendarbeit notwendigen Konzeptentwicklung, sowie als Ansatz einer Praxisforschung (Deinet, 2011).

Durch die empirisch begründeten Einblicke in subjektive Lebenswelten und das Erleben von Kindern in ihren Wohngebieten unter dem Aspekt der Raumaneignung wurden bspw. Verfahren einer sozialräumlichen Konzeptentwicklung entwickelt (Deinet \& Krisch, 2002). Aus der Beobachtung, Analyse und Interpretation des Raumaneignungsverhaltens von Kindern und Jugendlichen sind auf dieser Grundlage auch die Bedarfe für die Entwicklung von Konzepten und konkreten Angeboten der Kinder- und Jugendarbeit besser abzuleiten (vgl. auch Empfehlungen in Deinet, Szlapka, \& Witte, 2008).

Das Ziel der qualitativen Sozialraumanalyse ist es, Verständnis dafür zu entwickeln, wie die Lebenswelten Jugendlicher in engem Bezug zu ihrem konkreten Stadtteil oder ihrer Region, $\mathrm{zu}$ ihren Treffpunkten, Orten und Institutionen stehen und welche Sinnzusammenhänge, Freiräume oder auch Barrieren Jugendliche in ihren Gesellungsräumen erkennen (Krisch, 2009).

Besonders in der Forschung mit Jugendlichen ist es von zentraler Bedeutung, sich die häufig bestehende Wahrnehmung von Distanz zwischen Erwachsenen und Jugendlichen bezogen auf Macht und Ungleichheit in der Betrachtung kritisch zu reflektieren. Gerade bei der Erhebung empirischen Materials ist es unablässig, den wahrgenommenen ,großen Graben“ (,great gulf") zwischen Erwachsenen und Jugendlichen in ihren oftmals doch recht unterschiedlichen Erfahrungswelten kritisch zu reflektieren (Raby, 2007).

\footnotetext{
${ }^{1}$ Der Begriff ,,infosphere“ ist eine Wortneuschöpfung aus den 1970er Jahren. Er basiert auf dem Begriff „Biosphäre“ als Bezeichnung der lebenschöpfenden und sich rasch entwickelnden Regionen auf der Erde: „Minimally, infosphere denotes the whole informational environment constituted by all informational entities, their properties, interactions, processes, and mutual relations. It is an environment comparable to, but different from, cyberspace, which is only one of its sub-regions, as it were, since the infosphere also includes offline and analogue spaces of information. Maximally, infosphere is a concept that can also be used as synonymous with reality, once we interpret the latter informationally. In this case, the suggestion is that what is real is informational and what is informational is real“" (Floridi, 2014, S. 54).
} 
Wenn also ein Verständnis von Lebenswelt und Lebensraum als Sozialraum von so großer Bedeutung ist, müssen wir uns fragen, mit welchen Methoden wir in der Sozialen Arbeit diese Welten und Räume empirisch erfassen können? Die Antwort auf diese Frage hat sich in der Literatur - und zunehmend auch in der Praxis - weitgehend auf zwei Aspekte verdichtet: die Bedeutung von Netzwerken als Manifestation der Lebenswelten und Lebensräume von Menschen und deren Sozialkapital innerhalb dieser Räume. Lassen Sie mich daher im folgenden auf diese beiden Dimensionen näher eingehen.

\subsection{Digitalisierung und soziale Beziehungen im digitalen Kapitalismus}

Seit seiner Einführung besonders durch die Werke von Schiller (1999) ist der Begriff „,digitaler Kapitalismus“ (Fuchs \& Mosco, 2016; Staab, 2016; Wajcman, 2015) bislang noch mit keiner einheitlichen und allgemein anerkannten Definition verbunden. Thrift (2005) etwa nutzt den Begriff „knowing capitalism“, der Begriff „Plattformökonomie“ (Srnicek, 2017) hat sich ebenso fest etabliert wie etwa Bezeichnungen wie „Postkapitalismus“ (Mason, 2015) oder „,new capitalism“ (Sennett, 2007). Dieser letzte Strang, beheimatet in vielen verschiedenen Literaturen, stellt nicht auf eine Weiterentwicklung kapitalistischer Strukturen, sondern auf deren Verschwinden in einer durch digitale Technologien dominierten Welt (beispielhaft etwa ElderVass, 2016).

Einige zentrale verbindende Elemente sind jedoch deutlich erkennbar. Zum einen versuchen die Autor*innen die historisch bekannte und gut erforschte transformative Bedeutung von Technologien auf die systematischen Formen —- der „Gestalt“ — des Kapitalismus in ihrer Analyse einzubeziehen und stellen dabei die besondere Rolle von digitalen Technologien für die Umwälzungen in Produktion, Dienstleistungen, der Arbeitswelt und den neuen Klassenformen (im Sinne einer Beziehung zwischen Kapital und Arbeit) heraus (Beer, 2019). Ein weiteres Element ist die besondere Rolle von Information und Daten (Gorz, 2010; Rullani, 2011; Smith, 1984), deren Bedeutung sich durch ihre Erweiterung der Erstellung, Sammlung und Verarbeitung in digitaler Form noch einmal wesentlich zentraler darstellt als schon in der Vergangenheit (Auerswald, 2017; Mayer-Schönberger \& Ramge, 2018). Ein dritter wesentlicher Fokus ist auf die Veränderung von Arbeit gerichtet (Baldwin, 2019; Ford, 2015; Fuchs, 2014; Herzog, 2019; Paus \& Ford, 2018; Zhang, 2018).

Im Rahmen dieses Textes wird digitaler Kapitalismus als eine transformation systemischer Strukturen in der Evolutionsgeschichte und -entwicklung des Kapitalismus als organisatorische Logik sozial-ökonomischer Systeme verstanden. Diese Entwicklung ist maßgeblich geprägt von der prägenden Rolle von Technologien und den Besitz- sowie Kontrollverhältnissen, welche diese Systeme prägen, insbesondere bezogen auf das Verhältnis von Arbeit und verschiedenen Formen von Kapital. ${ }^{2}$

Zuboff (2018) stellt die herausragende Bedeutung von Daten in diesem neuen System dar. Sie bezeichnet diese Erweiterung des kapitalistischen Systems als „Überwachungskapitalismus" (surveillance capitalism). Sie weisst dabei auf drei wesentliche Aspekte hin, welche diese Form des Kapitalismus von Vorgängerformen unterscheidet: (1) die Forderung nach unreglementierter Freiheit und „Wissen“ (knowledge) im Sinne gesammelter Informationen; (2) einen

\footnotetext{
${ }^{2}$ Ich folge hier im wesentlichen den Ausführungen von Kocka (2014). Dieser verweist auf (1) individuelle Eigentumsrechte; (2) Koordinierung der verschiedenen wirtschaftlichen Akteure vor allem über Märkte und Preise, Wettbewerb und Zusammenarbeit, über Nachfrage und Angebot bzw. Verkauf von Waren; (3) Kapital als grundlegendes Element dieser Koordinierung und Zusammenarbeit (Kocka, 2014, Kapitel I, 3, 6. Absatz).
} 
Bruch mit der Akzeptanz der Notwendigkeit eines - wenngleich ungleich im Machtverhältnis - reziproken Beziehungssystems zwischen Kapital und dem Datenproletariat; und (3) einer Neuordnung der kollektivistischen Ordnung und Orientierung hin zur Trennung von Staat und privater Macht des Kapitals, welche den Staat nicht mehr als Ordnungsmacht in einer symbiotischen Beziehung benötigt, sondern Wissen (knowledge) privatisiert und somit Kontrolle weitgehend unabhängig von staatlicher Ordnung ausüben kann (vgl. auch Dijck, 2014). ${ }^{3}$

Diese Entwicklung wird begünstig durch ein Interesse an interpersonalen Daten nicht nur von privatwirtschaftlicher Seite, sondern eben gerade auch einerseits von staatlicher, als auch von akademischer Seite:

In fact, all three apparatuses — corporate, academic, and state-are highly staked in getting unrestraint access to metadata as well as in the public's acceptance of datafication as a leading paradigm. Scientists, government agencies and corporations, each for different reasons, have a vested interest in datafied relationships and in the development of methods that allow for prediction as well as manipulation of behavior. (Dijck, 2014, S. 203)

Somit besteht aus zum Teil recht unterschiedlichen Interessenlagen ein starker Anreiz der wesentlichen institutionellen Akteure die bestehenden Vertrauensstrukturen auf die Neuordnung gesellschaftlicher Strukturen im digitalen Zeitalter zu übertragen. Dabei kommen durch ihre Kompetenzen und den ihnen zur Verfügung stehenden Ressourcen diesen Institutionen eine herausragende Stellung und Verantwortung in der gesamtgesellschaftlichen Debatte zu: dem Staat als Kontrollinstanz, der Privatwirtschaft durch Innovationen von Technologien und Prozessen und der Wissenschaft in ihrer Doppelfunktion als Grundlagenforschung und kritischreflektive Forschung betreibendes Bindeglied aller beteiligten Akteure.

Die besondere Bedeutung der zentralen Stellung von Daten in einem digitalen kapitalistischen System drückt sich etwa in der Umkehr der Beziehung zwischen „Erinnerung und Vergessen“ wider:

For millennia, the relationship between remembering and forgetting remained clear. Remembering was hard and costly, and humans had to choose deliberately what to remember. The default was to forget. In the digital age, in what is perhaps the most fundamental change for humans since our humble beginnings, that balance of remembering and forgetting has become inverted. Committing information to digital memory has become the default, and forgetting the exception. (Mayer-Schönberger, 2011, S. 196)

Gerade für schutzbedürftige Gruppen in der Gesellschaft stellt dieser Wandel eine besondere und zunehmende Herausforderung dar, denn er stellt zum einen eine weitere signifikante

\footnotetext{
${ }^{3}$ Diese Forderung von Seiten des Kapitals schliesst eine eigenständige Sammlung und Nutzung von Wissen und Kooperation mit privaten Akteuren von Seiten des Staates keineswegs aus, institutionalisiert in Form von Organisationen wie der US-amerikanischen NSA die es vergleichbar bei allen staatlichen Akteuren gibt (Baumgärtel, 2013), aber auch weit über den Sicherheitsbereich in einer Neuformation staatlich-bürokratischen Handelns sichtbar wird (Muellerleile \& Robertson, 2018).
} 
Machtverschiebung hin zu Datenbesitzenden, ${ }^{4}$ zum anderen erfüllt „Vergessen“ eine wesentliche Funktion für menschliche Handlungsentscheidungen, da es Menschen erlaubt mit Abstraktionen und Generalisierungen als mentale Brücken in der Entscheidungsfindung zu arbeiten und Wandel als Bestandteil menschlicher Entwicklung zu berücksichtigen (Mayer-Schönberger, 2011, S. 197).

Diese Entwicklung spiegelt sich auch in der zunehmenden Bedeutung privat-rechtlicher Klassifizierungen wider, deren Hintergrund und Entstehung für Betroffene meist nicht einsehbar und nur schwierig beeinflussbar sind, jedoch analog zu Klassenzugehörigkeit im Weberschen Sinne für die Handlungsoptionen von Menschen in modernen Gesellschaften mittlerweile ganz massgeblich geworden sind (Fourcade \& Healy, 2013). Sevignani (2017) in seiner Diskussion dieser Entwicklung betont daher nicht nur die Zusammenhänge zwischen Klassifizierung und soziologischer Manifestation bestehender Klassenzugehörigkeit, sondern auch auf den Umstand, dass die durch die Digitalisierung noch beschleunigte Entwicklung der Informationalisierung (informatisation) mittlerweile die enge Verknüpfung und Modellierung aller Stufen des ökonomischen Prozesses erlaubt (Sevignani, 2017, S. 79).

Die folgenden Abschnitte sollen die Bedeutung der durch den digitalen Kapitalismus hervorgerufenen Veränderungen in Bezug auf wichtige theoretische soziologischen Konzepte die im Rahmen der Sozialen Arbeit Verwendung finden — dem Sozialraum, dem Konzept des Vertrauens (Trust) und Sozialkapitals, sowie der Konstitution und Bedeutung von Gruppen — und deren methodischen Erfassung und Analyse kurz skizzieren.

\subsection{Sozialraum im digitalen Kapitalismus}

Das Konzept der Raumaneignung als ein wesentliches Kennzeichen der sozialraumorientierten Sozialen Arbeit macht diesen Ansatz besonders wertvoll in der methodischen Reaktion auf die transformatorischen Veränderungen der Lebenswelten von Menschen in einer zunehmend von Globalisierung und Digitalisierung geprägten Lebensrealität. Soziale Räume schliessen eben zunehmend auch virtuelle Räume mit ein, welche eine Grenzziehung der „Räumlichkeit" — ohnehin immer eine besondere Herausforderung in der empirischen Erhebung (s. etwa Brandtzaeg \& Lüders, 2018) - und die erforderlichen Methoden der Erforschung der Aneignungsprozesse zu einer stetigen analytischen Herausforderung machen, gerade in der praktischen Anwendung innerhalb der Sozialen Arbeit. Diese Entwicklung umfasst ausdrücklich nicht nur ausschliesslich oder primär ,digital“ dominierte Räume — etwa online mediatisierte Gruppen- und andere soziale Beziehungen — sondern mittlerweile fast alle sozialen Beziehungsräume und Bereiche (vgl. Cortada, 2012; BMG, 2017; OECD, 2019b; Paus \& Ford, 2018; Skutta \& Steinke, 2019).

Die Veränderungen der durch „Social Media“ hervorgerufene Erweiterung des Sozialraums (Berezan, Krishen, Agarwal, \& Kachroo, 2018; McInroy \& Craig, 2018) oder den Herausforderungen der Transnationalität in einer von digitalen Medien und Technologien geprägten

\footnotetext{
${ }^{4}$ Die Entwicklung setzt einen Prozess fort, der seinen Ursprung in den ersten schriftlichen Urkunden fand (Ifrah, 2000; Menninger, 1958; Wright, 2007), insbesondere auch in der Form der Kartographie (Branch, 2011; Brotton, 1998) und seit dem 18. Jahrhundert noch einmal eine weitere entscheidende Erweiterung erfuhr (Behrisch, 2006b). Dabei stellt der eigentlich „Besitz“ der Daten nur einen Aspekt dar; für die Entstehung von Macht aus diesen Daten heraus ist vielmehr entscheidend die mit dem Prozess der Informationalisierung (informatisation) und Datifizierung (datafication) verbundene Kodifizierung und Analyse von Daten und der Verbindung mit Personen und Institutionen. Vgl. auch weitere Ausführungen später unter 2.3 Vertrauen im digitalen Kapitalismus und 3 Kompetenzen für die Soziale Arbeit in einer digitalisierten Welt: Sprachfähigkeit und Methoden.
} 
Migrationsgesellschaft wie der unsrigen (Dey, Balmer, Pandit, \& Saren, 2018; Schneider, 2018) machen deutlich, wie wichtig die Einbeziehung nicht-physisch begründeter Räume und Netzwerke in die Konzeption des Sozialraums in allen Lebensbereichen schon jetzt geworden ist. Diese Entwicklung verstärkt sich mit der zunehmenden Hybridisierung von sozio-ökonomischen Beziehungen weiter (OECD, 2019a).

\subsection{Vertrauen im digitalen Kapitalismus}

Definiert als zuversichtlich-positive Erwartung bezogen auf das Handeln anderer (Lewicki, McAllister, \& Bies, 1998) stellt Vertrauen nach Giddens (1990) ein zentrales Element für die erneute „Modernisierung der Moderne“ und in der Formen der Mechanismen von Vertrauen in abstrakte Systeme ein konstituierendes Element moderner Institutionen dar. Die Möglichkeit der Entstehung von und das Maß an Vertrauen zwischen Subjekten und Gruppen stellt somit ein wesentliches Fundament menschlichen Miteinanders in allen sozialen Beziehungen dar (Sztompka, 1999), indem es die Komplexität insbesondere moderner Lebensbezüge reduziert und überhaupt erst ermöglicht (Earle \& Cvetkovich, 1995, S. 38; vgl. auch Luhmann, 1968/2014; Coleman, 1994; Gambetta, 1988).

Insbesondere in einem von einer sprunghaft ansteigenden Datifizierung (datafication) gekennzeichneten Umfeld (Galliers, Newell, Shanks, \& Topi, 2017; Holtzhausen, 2016; Marjanovic \& Cecez-Kecmanovic, 2017) stellt Vertrauen somit als Grundlage menschlichen Miteinanders ein sich in seiner institutionellen Form stark verändertes und weiterhin veränderndes elementares Gut dar. In seiner Bedeutung als komplexitätreduzierendes Mittel der Ermöglichung von Beziehungen gewinnt es durch die stark gestiegene Komplexität von Lebensbezügen in rein digitalen und hybriden Formen immens an Bedeutung (@ Dutton \& Shepherd, 2006; Madhavan \& Wiegmann, 2007). Somit ersetzen beispielsweise Online Bewertungen unbekannter Personen den Rat von Personen aus dem eigenen unmittelbaren Bezugsumfelds - eine uns alle mittlerweile vertraute Form der Ratifizierung von Beziehung und Vertrauen, oftmals sogar in zum überwiegenden Teil rein quantitativer Form auf einer Werteskala (Kollock, 1999; Lee \& Turban, 2001; Lunn \& Suman, 2008).

Dijck (2014) hebt die besondere Bedeutung von Vertrauen nicht nur bezogen auf das Handeln spezifischer staatlicher Institutionen oder einzelner wirtschaftlicher Akteure hervor, sondern bezogen auf die Rechtmässigkeit des gesamten digitalen Ökosystems. Dabei kommt, analog zu Althusser (1971), dem „Dataismus“ (dataism) die Funktion der Interpellation zu - also der Subjektivierung des Individuums durch Daten - bezogen auf gemeinsame Überzeugungen einer „Rechtsstaatlichkeit“ die auf institutionellem Vertrauen basieren. Nach Raley (2013) nimmt hinsichtlich der umstrittenen Wahrnehmung von „Vertrauen“ und „Überzeugung“ (belief) die gesellschaftliche Debatte über „Dataveillance“5 als bevorzugte Form der staatlichen Überwachung durch soziale Medien und onlinebasierter Kommunikation eine immer wesentlichere Stellung ein:

In this respect, making data public is also making a commons. Apart from functioning as a rival form of expertise, then, one effect of these countervailing tools and techniques has been to re-embed dataveillance within social relations. (Raley, 2013, S. 133)

\footnotetext{
${ }^{5}$ Der Begriff „dataveillance“ ist eine Verbindung der Begriffe „,data“ und „surveillance“, also einer starken sozialen Überwachung auf Basis der (teil)automatisierten Observation durch (Meta)Datensammlung und -analyse (Clarke \& Greenleaf, 2017).
} 
Die Öffentlichmachung von Daten kreiert also sowohl eine neue gesamtgesellschaftliche Öffentlichkeit als auch eine neue Allmende als gemeinsamen Ort des Handelns von Subjekten sowohl im realen, als auch im virtuellen Bereich.

\subsection{Sozialkapital im digitalen Kapitalismus}

Soziales Kapital (Burt, 2005; Lin, Cook, \& Burt, 2001) wird im Wesentlichen von drei Merkmalen gekennzeichnet:

1. Intensität der Beziehung

2. Strukturgeschlossenheit

3. Zeitgeschlossenheit

Im europäischen Kontext hat insbesondere Pierre Bourdieu den Begriff des sozialen Kapitals geprägt. Er beschreibt dieses als ,die Gesamtheit der aktuellen und potenziellen Ressourcen, die mit der Teilhabe am Netz sozialer Beziehungen gegenseitigen Kennens und Anerkennens verbunden sein können. Es handelt sich dabei um Ressourcen, die auf der Zugehörigkeit zu einer Gruppe beruhen“ (Bourdieu, 1983, S. 190f). Der Umfang des Sozialkapitals einer Person hängt sowohl von der Größe als auch von der Qualität (der Intensität des Austauschs und der Macht der Netzwerkpartner) des sozialen Netzwerkes ab. Die Mitglieder sozialer Netzwerk weisen eher ähnliche Charakteristika auf, und zwar nicht nur ähnliche sozioökonomische Positionen, sondern auch ähnliche Wertvorstellungen und Verhaltensmuster, also dem Bourdieuschen Habitus.

Im Unterschied hierzu steht die Sichtweise von Coleman (1988); hier sind vor allem die konkreten Beziehungen zwischen einzelnen Akteuren von Interesse und wie über eine Verstetigung und qualitative Umformung bloßer Kontakte zu einer gegenseitigen Vernetzung ein Mehrwert - ein besonderes soziales Kapital — für alle Beteiligten entsteht. Dies basiert weitgehend auf Granovetter's (1985) Konzept der Eingebundenheit (,embeddedness“).

Nach Coleman (1988) ist soziales Kapital eine aus sozialen Beziehungen erwachsende Ressource, deren Nutzen über verschiedene Formen vermittelt ist, u.a. über

- gegenseitige Verpflichtungen und Erwartungen (,wie Du mir, so ich Dir“);

- einem leichteren und besseren Zugang zu Informationen als Handlungsgrundlage;

- die Garantie von Normen und Sanktionen, auf die sich gegenseitig verlassen werden kann; und

- multiplexe Beziehungen, in denen Ressourcen aus einer Beziehung für andere Beziehungen genutzt werden.

Darüber hinaus kann soziales Kapital auch über etablierte Herrschaftsbeziehungen, die die Verantwortung für Kontroll- und Gewährleistungsaufgaben arbeitsteilig ausgestalten, oder über soziale Organisationen und deren strukturiert-zielgerichtete Tätigkeiten vermehrt werden.

Eine weitere Definition von Sozialkapital in einer expliziten Erweiterung von Gesellschaft über den physischen Begegnungsraum hinaus für die sog. Wissensökonomie (knowledge economy) findet sich etwa bei Westlund: 
Social capital is defined as social, non-formalized networks that are created, maintained and used by the networks' nodes/actors in order to distribute norms, values, preferences and other social attributes and characteristics, but which also emerge as a result of actors sharing some of these attributes. (Westlund, 2006, S. 7)

Ein wichtiges Element dieser Definition ist die Unterscheidung zwischen Netzwerken und Normen, die in diesen Netzwerken transportiert werden bzw. oder auf denen die Netzwerke selber basiert sind. Sozialkapital in dieser Sicht ist eine Art Infrastruktur zwischen Knoten (nodes) - also Akteuren wie einzelnen Individuen aber auch Organisationen - und Kanten (links), den Verbindungen zwischen diesen Akteuren. Die Entstehung dieser Verbindungen wird dabei gesteuert von den Normen, Präferenzen und Haltungen (attitudes).

Die teils massiven transformatorischen Prozesse die unsere heutige soziale Realität prägen Globalisierung, Migrationsgesellschaft, Digitalisierung, steigende soziale Ungleichheit, um nur einige wenige zu nennen — stellen Professionelle Akteure in der Sozialen Arbeit vor neue Herausforderung, den Sozialraum auch in der Zukunft in seiner Ausgestaltung und Konkretisierung als Erlebniswelt begleiten und analytisch erfassen zu können (Häußling, 2010). Gerade die Messung von starken und schwachen sozialen Beziehungen (social ties) ist mittlerweile eine anerkannte und ausdifferenzierte Methode um Sozialkapital und seine Bedeutung für einzelne Akteure (Mikro) aber auch das gesamte betrachtete System (Makro) empirisch zu betrachten und $\mathrm{zu}$ analysieren (Marbach, 2010). Alle diese Veränderungen stehen auch in einem engen Zusammenhang miteinander. Im folgenden Abschnitt will ich mich in der gebotenen Kürze im Kontext dieses Textes auf den Prozess der Digitalisierung konzentrieren und den damit verbundenen Transformationsprozess bezogen auf unsere gesamtgesellschaftlichen Sozialsystem skizzieren.

Aber auch die Bedeutung von Sozialkapital verändert sich durch diese Entwicklungen und auch hier wieder durch die zunehmende Bedeutung von Social Media in der Gestaltwerdung von Lebenswelten - gerade von jungen Menschen (Buckingham, 2008). Wir kennen die Beispiele von jungen Menschen in sozialen Netzwerken, die als sog. „Influencer“ ganz massgeblich Einfluß auf Lebenswelten und interpersonale soziale Netzwerke nehmen (Abidin \& Brown, 2018; Guerrero-Pico, Masanet, \& Scolari, 2018) aber auch die Problematik von Cyberbullying (Betts, 2016; Li, Cross, \& Smith, 2012; Slonje \& Smith, 2008; Smith u. a., 2008). Beide Beispiele sind eng mit der Veränderung der Manifestation von „Kapital“ in sozialen Beziehungen verbunden, sobald digitale Technologien eine Rolle spielen. Diese Rolle nimmt stetig in der Lebensweltrealität junger Menschen zu, in bewußten (Kontaktmedien) und unbewußten (Algorithmen, Datenpfade) Formen.

\subsection{Gruppen in einer digitalisierten Welt}

Lassen Sie uns diese Entwicklungen an einem gut vertrauten und bewährten soziologischen Konzept reflektieren: dem Konzept der Gruppe. Das Konzept der Gruppe als ein wesentliches Element von identifikationskonstituierenden Prozessen scheint so allgegenwärtig und selbstverständlich, dass wir Gruppen leicht als grundlegende Elemente sozialen Lebens, als wesentliche Akteure sozialer Konflikte und als analytische Grundeinheit in der Analyse einfach als gegeben voraussetzen (vgl. Brubaker, 2006, S. 2). Nimmt man aber die Soziologie der Gruppe und die Diskussionen in der Literatur ernst, ist zunächst immer erst empirisch nachzuweisen, wann, wo und wie Gruppen in der Wahrnehmung und Handlung von Menschen — sprich außerhalb oder 
innerhalb ihrer vermeintlichen Gruppenbezüge - relevant werden. Gruppen konstituieren und reproduzieren sich oder verfallen durch Handlungsvollzüge und nicht jenseits dieser (Barth, 1969).

Will man also Gruppen bzw. Zugehörigkeiten zu Gruppen nicht nur zuschreiben, sondern Prozesse der Gruppenbildung empirisch identifizieren, reflektieren und als Teil der Sozialen Arbeit nutzen - und zwar dort, wo sie sich ,im Denken, Fühlen und Handeln von Menschen allgemein und Kindern und Jugendlichen im Besonderen manifestieren“ (Skrobanek, 2016, S. 118) - dann stellt sich die Frage: reichen unsere bestehenden Methoden und Modelle dafür aus in einem von Digitalisierung und hybriden Beziehungsmustern geprägten sozialen Realität? Wenn Fühlen und Handeln sich zumindest in Teilen immer stärker auch in digitalen Räumen manifestiert: droht durch diese Entwicklung der ,great gulf“ zu einem ,,even greater gulf“ zwischen Jugend und Erwachsenen-Erfahrungswelten in unserer Ausbildung Sozialer Arbeiter*_ innen zu werden? Sind diese in der Lage, auch zukünftigen Weiterentwicklungen dieser sich verstärkenden Prozesse analytisch-methodisch zu begegnen?

\section{Kompetenzen für die Soziale Arbeit in einer digitalisierten Welt: Sprachfähigkeit und Methoden}

Die im vorigen Abschnitt aufgeführte gestiegene Bedeutung von Daten als Resultat einer Datafizierung im gesamtgesellschaftlichen Miteinander macht es notwendig, über die Kompetenzen zu sprechen, die für eine zeitgemäße, professionelle und unterstützende Soziale Arbeit jetzt und in der Zukunft erforderlich sind. Wenden wir unseren Blick daher kurz auf Daten als Rohstoff und die Grundlage eines zunehmend digitalisierten Sozialraums. Daten verstanden als jede manifeste (real oder abstrakt) Merkmalsausprägung (Variable, Attribut) eines bestimmten Merkmalsträgers (Personen, Objekte, Ereignisse) — werden in ihrer Form häufig u.a. in zwei grundsätzliche Typen ${ }^{6}$ unterschieden: strukturierte und unstrukturierte Daten (vgl. Kelleher \& Tierney, 2018, Kapitel 2). Strukturierte Daten sind solche Daten, die in ihrer Ausprägung gleichermassen strukturiert sind und in einer Tabelle festgehalten werden können (Name, Geburtsort, Alter, Ausbildungsgrad). Unstrukturierte Daten hingegen sind Daten, bei denen jeder Merkmalsträger eine eigene interne Struktur aufweist die nicht zwingend mit der Struktur anderer Merkmalsträger übereinstimmt (beispielsweise Briefe von Personen oder Dokumente).

In der methodologischen Literatur wird häufig auch zischen „,Rohdaten“ und ,,abgeleiteten Daten“ unterschieden: Rohdaten (raw data) sind in Daten „direkt gemessen“ und nicht in irgendeiner Form transformiert wurden oder aus anderen Daten indirekt neu gebildet wurden, dem Merkmal von abgeleitet Daten (derived data) (Kitchin, 2014, S. 34). Während in der Vergangenheit gerade in den Sozialwissenschaften die abgeleiteten Daten häufig eine zentrale Stellung einnahmen, ändert sich dieser Trend durch die Datafizierung nun zunehmend, da durch neue Methoden Daten in Form von rohdatenbasierten „Big Data“"7 (De Mauro u. a., 2015) Daten-

\footnotetext{
${ }^{6}$ In Statistiktextbüchern wird häufig auch auf der Basis der Typenart von Daten unterschieden, also numerisch, nominal und ordinal. Genau so gut werden Daten häufig auch auf Grundlage ihrer methodischen Erhebungsform unterschieden. Grundsätzlich lässt sich festhalten (Kitchin, 2014, S. 27): „In broad terms, data vary by form (qualitative or quantitative), structure (structured, semi-structured or unstructured), source (captured, derived, exhaust, transient), producer (primary, secondary, tertiary), and type (indexical, attribute, metadata).“

${ }^{7}$ De Mauro, Greco, \& Grimaldi (2015) bieten folgende formale Definition des Begriffs an: „Big Data represents the Information assets characterized by such a High Volume, Velocity and Variety to require specific Technology
} 
sätzen auch in den Sozialwissenschaften analytisch an Bedeutung gewinnen (Chen, 2018; Foster, 2017; Günther, Rezazade Mehrizi, Huysman, \& Feldberg, 2017; Rudder, 2014; Taylor \& Broeders, 2015), wenngleich diese Entwicklung durchaus kritisch hinterfragt wird (Lane, Stodden, Bender, \& Nissenbaum, 2014; Markus, 2017).

Ganz entscheidend für diese Entwicklung sind die sog. „Abgasdaten“ (exhaust data), die zwar gleichermassen Rohdaten sind, sich jedoch von den abgeschöfpften Daten (captured data) darin unterscheiden, dass sie gewissermassen als Nebenprodukt des regulären Abschöpfungsprozesses entstehen. Insbesondere Metadaten, also Daten die andere Daten beschreiben, nehmen hier eine zentrale Stellung ein (Kelleher \& Tierney, 2018, Kapitel 2.1, Abs. 3). Durch die Methoden der Big Data Analyse gewinnen diese Metadaten eine enorme Bedeutung in der Erforschung und Analyse von Verhaltensmustern, die bislang nur verzerrt, indirekt oder überhaupt nicht gemessen werden konnten und entweder auf Eigenaussagen oder Beobachtung basierten.

Daten so verstanden sind jedoch niemals wirklich in „Rohform“ existent, sondern gewinnen immer nur Bedeutung in einem sozialen Kontext (Gitelman, 2013). Akteur*innen der Sozialen Arbeit nehmen als Teil ihrer Arbeit auch eine wesentliche Anwaltschaftsrolle für Personen und Gruppen ein, deren Teilhabe in einer gesamtgesellschaftlichen Debatte keineswegs gesichert und gleichberechtigt neben derer anderer Gruppen steht. Hierfür ist die eigene Fähigkeit von Akteur*innen der Sozialen Arbeit der aktiven Teilnahme in gesellschaftlichen Debatten Grundvoraussetzung. Diese aktive Teilnahme bedingt heute zunehmen eine Sprachfähigkeit, die über die klassischen Formen der textbasierten Kommunikation von Wissen und Interessen hinausgeht (Baack, 2015). Ebenso ist für eine Unterstützung von Klient*innen zur Befähigung einer aktiven, gesamtgesellschaftliche Teilhabe eine eigene digitale und datenbezogene Sprachfähigkeit essentiell. Daraus erwächst für die Soziale Arbeit ein neues Anforderungsprofil in der Lehre auf die ich später näher eingehen werde.

Abbildung 1 zeigt schematisch den Zusammenhang zwischen den Elementen der Datenzentralität - der Erstellung von Daten, der Sammlung von Daten und der Analyse von Daten — und den daraus entstehenden Herausforderungen — dem Umgang mit verschiedenen und oftmals konkurrierenden Normen, der Kodifizierung von qualitativem Wissen und den damit verbundenen Interessen in Form von Daten, sowie der Fähigkeit, Daten und digitale Formen der Kommunikation zu ver- und entschlüsseln und sich aktiv in diesen an gesellschaftlichen Diskursen zu beteiligen. Diesen Herausforderungen muss Lehre und Ausbildung der Sozialen Arbeit gerecht werden, wenn sie Studierende mit den notwendigen Kompetenzen für ein professionelles Handeln in der Sozialen Arbeit befähigen möchte. Die Digitalisierung verstärkt den schon seit einiger Zeit bestehenden Trend zur Datenzentralität von gesellschaftlichen Diskursen.

Qualitatives Wissen wird oftmals primär in quantitativer Form kodifiziert und in digitalen Formaten gespeichert. Dieser Prozess stellt ein wesentliches Merkmal sozio-ökonomischer Entwicklung seit Millennia dar (Auerswald, 2017; Ifrah, 2000; Wright, 2007) und hat seine neueren historische Wurzeln im 18. Jahrhundert und der europäischen Moderne. Seit dieser Zeit wurden Maß und Zahl zu entscheidenden Kategorien der Wahrnehmung, vor allem aufgrund ihrer bestechenden Evidenz und Präzision als scheinbar objektive Maßeinheiten politischen Handelns und zu idealen Medien der politischen Kommunikation (Behrisch, 2006a, S. 7). Während dieser Prozess anfangs dem Staat eine ,zunehmende innere Herrschaftsverdichtung“ (Behrisch, 2006a, S. 7) und damit die Erlangung zentraler Kontrollfunktionen über individuelles Handeln ermöglichte, haben diese Kontrollfunktion zunehmend private Akteure übernommen, deren Interessen hauptsächlich auf kommerzielle Interessen ausgerichtet sind (Beer, 2019;

and Analytical Methods for its transformation into Value“ (S. 103). 


\section{Datenzentralität}

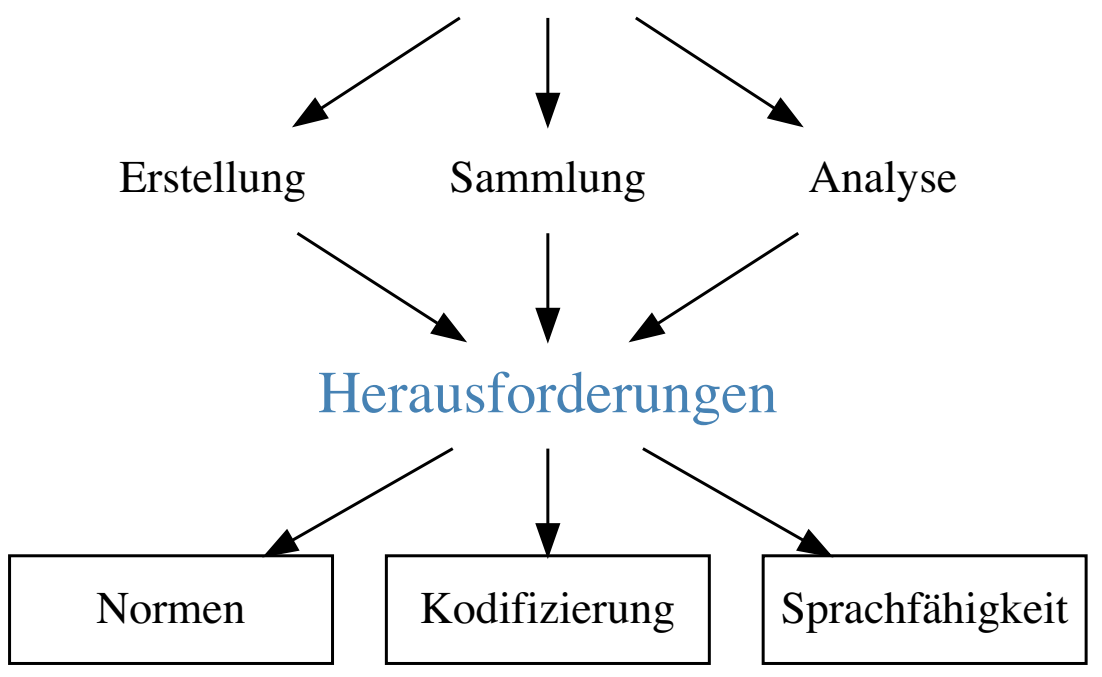

Abbildung 1: Schematische Darstellung Zusammenhang von

Datenzentralität und Kompetenzherausforderungen

Sennett, 2007; Thrift, 2005).

Dementsprechend sind wesentliche gesamtgesellschaftliche normative Werte - und somit die Grundlage von Bewertungsmustern und -rastern - in Zahlen kodifiziert und manifestieren sich immer häufiger in Abstraktionen, die sich für eine zunehmende Zahl von Betroffenen nicht immer eindeutig erfassen und nachvollziehen lassen. Die Digitalisierung verstärkt diese Entwicklung noch weiter hin zum einer Situation, in der Handlungen oftmals durch Daten bewusst manipuliert werden, ohne dass dieser Prozess den Handelnden überhaupt bewusst ist oder analysiert und kritisch hinterfragt werden kann (Wu, 2017; Zuboff, 2018).

Der Umfang dieser Arbeit erlaubt es nur Teilaspekte der notwendigen Kompetenzen für die Soziale Arbeit in einer digitalisierten Welt zu beleuchten. Im folgenden werde ich mich daher auf lediglich zwei Bereiche beschränken: (1) der sog. „Sprachfähigkeit“ (im englischen: „literacies") in den Bereichen digitale und Datenkommunikation, sowie (2) der erweiterten Methodenkompetenz einer etablierten Analysemethode der Sozialen Arbeit, der Netzwerkanalyse.

\subsection{Sprachfähigkeit in digitaler und Datenkommunikation}

Für die aktive und gelungene Teilhabe in der Gesellschaft ist die „Sprachfähigkeit“ — also die Kompetenz, Informationen über Texte aufzunehmen und zu verfassen und dies als Wissen zu rezipieren, im englischen „literacy“ - notwendige und zwingende Voraussetzung. Dies gilt umso mehr in einem sich veränderten sozialem Umfeld, welches von neuen Formen der Literacy geprägt ist und insbesondere die Notwendigkeit voraussetzt, sich zukünftig in einer von digitalen Technologien dominierten Umwelt bewegen zu können (Cope \& Kalantzis, 2000; Jenkins, 2009; Lankshear \& Knobel, 2008).

Martin etwa macht einen direkten Bezug von Literacy auf den Erwerb notwendigen Sozialka- 
pitals für Teilhabe, wenn er feststellt:

[T] he idea of literacy expresses one of the fundamental characteristics of participation in society. (Martin, 2008, S. 155)

Bezogen auf die Operationalisierung und Messung werden allgemein drei Hauptdomänen der Sprachfähigkeit (literacy skills) unterschieden (OECD, 2000, x):

- Prosa-Verständnis (prose literacy): notwendige Kenntnisse und die Fähigkeit, Informationen aus allgemeinen Texten (Medien, Broschüren, Handbücher etc.) zu verstehen und anzuwenden.

- Dokumenten-Verständnis (document literacy): notwendige Kenntnisse und die Fähigkeit, Informationen aus Ausschreibungen, Formularen, Listen etc. zu identifizieren, zu rezipieren und deren Inhalte anzuwenden.

- Quantitatives Verständnis (quantitative literacy): notwendige Kenntnisse und die Fähigkeit, mathematische Operationen auf Zahlen in Texten anwenden zu können.

Alle drei Domänen erfahren durch die Digitalisierung von Medien und Informationen eine erhebliche Transformation bezogen auf die Form der Informationscodierung und kontextualisierung (Lange, 2014; Lankshear \& Knobel, 2011; Potter \& McDougall, 2017). Dies wiederum bedingt eine Erweiterung des Kompetenzprofils in allen drei Bereichen. Während in einer Print-Medien dominierten Medienkultur editoriale Bezüge der Information noch relativ überschaubar waren, sind diese in einer digital-medialisierten Umgebung wesentlich komplexer, bezogen auf Quellen, Inhalte und ein gesamtgesellschaftliches Gesamtnarrativ (Kramp, Novy, Ballwieser, \& Wenzlaff, 2013; Müller, Ligensa, \& Gendolla, 2009).

Gleichzeitig wächst aber der Einfluss der Medien auf soziale Systeme (Clark \& Slyke, 2010; Hattler \& Thomas, 2016; Howard, 2010). Wenn also die Erlangung dieser Kompetenzen für Empfänger*innen von Dienstleistungen der Sozialen Arbeit für eine gelingende gesellschaftliche Teilhabe immer zentraler werden, wird ein kompetenter Umgang mit und Kommunikation von und in Daten sowie der Fähigkeit der Vermittlung von Kernkompetenzen in der digitalen Kommunikation von Akteuren der Sozialen Arbeit Grundvoraussetzung für eine erfolgreiche Unterstützung und Begleitung (vgl. Otto \& Ziegler, 2010).

\subsubsection{Digital Literacy}

Aus der obigen Diskussion wird deutlich, dass „Digital Literacy“ als soziale Kompetenz gerade für die Soziale Arbeit immer wichtiger wird, wenn Klient*innen auf dem Weg zur Handlungsbefähigung in einer digitalisierten Umwelt begleitet und erfolgreich unterstützt werden sollen. Der Begriff ist keineswegs uniform definiert, beschreibt jedoch allgemein immer eine Erweiterung der Literacy (wie oben diskutiert) in die digitalen Domänen der Kommunikation und die Fähigkeit der Bedeutungsgebung (meaning-making) innerhalb und in Assoziation verschiedenen Modi i

Goodfellow (2011) hinterfragt den Begriff kritisch hinsichtlich der Frage, um welche Kernkompetenzen es denn spezifisch bei der Erweiterung der Begrifflichkeit der Literacy in Verbindung mit einer oftmals weitgehend unklar umrissenen Domäne des Digitalen geht, in dem er die 
Rekonzeptionalisierung des Begriffs Literacy in einer von digitalen Medien gekennzeichneten Welt nachzeichnet. Er stellt dabei besonders heraus, wie wichtig hierbei die Vermittlung der Fähigkeit von kritische Praktiken in der Ausbildung ist.

Digital Literacy so verstanden bedeutet also nicht nur (aber eben auch) die Fähigkeit eines kompetenten Umgangs mit digitalen Medien in der Kommunikation, sondern auch die Fähigkeit, verschiedene Wege der Bedeutungsgebung (meaning-making) auf der Grundlage digitaler und mit diesen Technologien verbundenen kulturellen Kodifizierungsschlüsseln zu beherrschen bzw. vermitteln zu können (vgl. auch Lankshear \& Knobel, 2008, und @lankshear-new-2006). Dabei ist es wichtig, gerade die Verbindung von bestehenden Textkompetenzen mit Kompetenzen aus der digitalen Domäne im Auge zu behalten und die Formung klassischer Literacies nicht zu vernachlässigen (Støle, 2018).

\subsubsection{Data Literacy}

Noch wichtiger wirkt sich die Transformation aus in den Domänen des Dokumenten- und quantitativen Verständnisses. Die zunehmende Quantifizierung sozialer Bezüge erfordert in der professionellen Arbeit auch in sozialen Bereichen ein tieferes Verständnis von Daten und Erweiterung des Kompetenzprofils bezogen auf deren Entstehung, Kontextualisierung, Interpretation und Präsentation als Information. Dieser Trend wird durch die Digitalisierung noch exponentiell verstärkt (Hintz, Dencik, \& Wahl-Jorgensen, 2018) und bedingt für professionelles sozialarbeiterisches Handeln eine erhebliche Erweiterung der Kompetenzen im Bereich der „Datensprachfähigkeit“(data literacy). Data Literacy ist die Fähigkeit, Daten auf kritische Art und Weise zu sammeln, zu handhaben, zu bewerten und anzuwenden (Ridsdale u. a., 2015) und dabei auch stets die regulativen und ethischen Normen im Blick zu haben. In einem Arbeitspapier über „Future Skills“ im Rahmen des Hochschulforums Digitalisierung stellen die Autor*innen fest:

Die Fähigkeit, planvoll mit Daten umzugehen und sie im jeweiligen Kontext bewusst einsetzen und hinterfragen zu können wird im Zuge der digitalen Transformationen von zunehmender Wichtigkeit und stellt eine zentrale Kompetenz in allen Sektoren und Disziplinen dar. Auf der einen Seite werden Data Scientists benötigt, die in der Lage sind, speziell mit großen heterogenen Daten umzugehen und die Technologie rund um den Big-Data-Lifecycle beherrschen, um schnell Entscheidungen basierend auf Daten und daraus abgeleiteten Informationen ermöglichen zu können. Auf der anderen Seite werden in der Breite in allen Sektoren und Disziplinen Personen benötigt, welche die Fähigkeit besitzen, Daten auf kritische Art und Weise zu sammeln, zu managen, zu bewerten und anzuwenden. Diese Fähigkeiten werden unter dem Begriff Data Literacy zusammengefasst. (Heidrich, Bauer, Iese, \& Krupka, 2018, S. 6)

Die sog. „Data Science“ (Skiena, 2017) versucht dieser Entwicklung Rechnung zu tragen, indem sie bislang weitgehend isolierte Methoden der Arbeit mit Daten interdisziplinär zu verbinden sucht. So weisen etwa Kelleher \& Tierney (2018) folgende Bereiche, graphisch in Abbildung 2 zusammengefasst, als Teil des Methodenkoffers von Datenwissenschaftler*innen aus: (1) Kommunikationskomptenzen, (2) Domänenwissen im Untersuchungsbereich, (3) ethisches und regulatives Wissen, (4) Kompetenzen in der Datenanalyse und im Umgang mit Datenbanken, (5) Informatikkompetenzen, (6) Datenvisualisierungskompetenzen, (7) Kompetenzen 


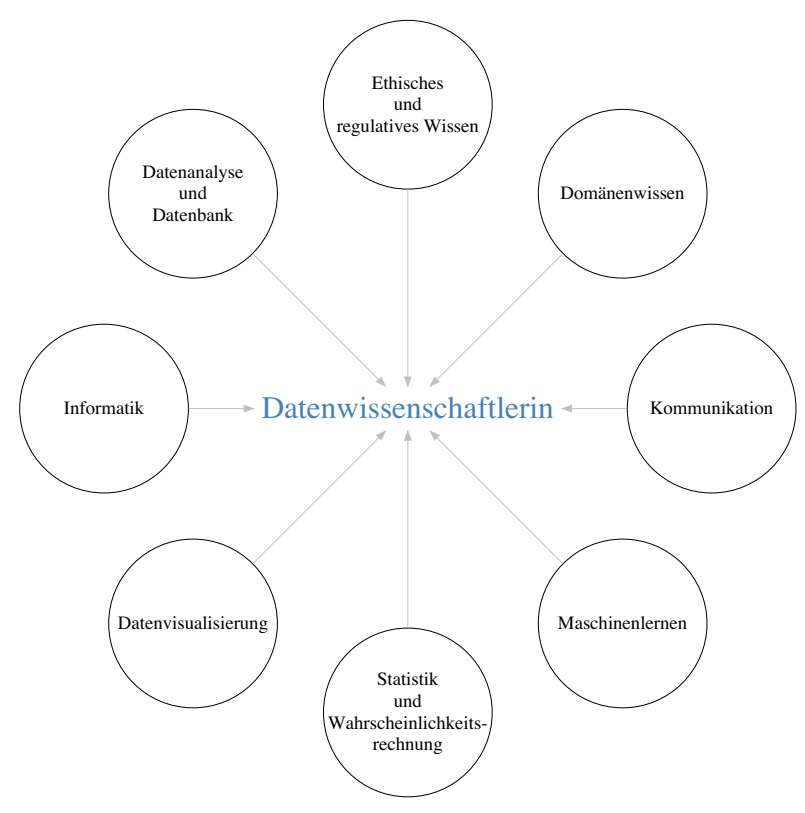

Abbildung 2: Kompetenzprofil einer Datenwissenschaftlerin, nach Kelleher \& Tierney (2018, Fig. 1)

in Statistik und Wahrscheinlichkeitsrechnung, sowie (8) Kompetenzen im Bereich Maschinenlernen.

Die Autoren machen deutlich, dass es dem oder der einzelnen Datenwissenschaftler*in kaum möglich ist, in allen diesen Bereichen eine tiefere Expertise zu besitzen. Wichtig ist jedoch ein grundsätzliches Verständnis aller Bereiche um die Möglichkeiten der Datenwissenschaft, die durch ihre Interdisziplinärität gekennzeichnet ist, voll ausschöpfen zu können. So ist es beispielsweise nicht zwingend notwendig, in der Ausbildung der Sozialen Arbeit eine tiefergehende methodische Informatikkompetenz oder im Bereich des Maschinenlernens zu erlangen. Wer jedoch erfassen möchte, wie Metadaten zur Kontrolle von sozialen Prozessen im öffentlichen Bereich genutzt werden und sich in der Debatte um die Ausgestaltung dieser Kontrolle und Mechanismen beteiligen möchte, der muss ein grundsätzliches Verständnis für die methodischen Grundlagen besitzen um an der gesellschaftlichen Debatte aktiv teilnehmen und eine Advokat*innenrolle für diejenigen Personen einnehmen zu können, welche die Tragweite und Formen der Kontrollmechanismen in ihrem Handeln oft nur sehr beschränkt wahrnehmen (können). Ebenso bedingt der verantwortliche Umgang mit datenbasierten Kontroll- und Steuermechanismen ein grundsätzliches Verständnis und Kenntnis über die Entstehung von datengestütztem Wissen insbesondere über Personen und deren Handlungen.

Der Rahmen dieser Arbeit lässt hier keine tiefergehende Darstellung der einzelnen methodischen Kompetenzen zu. Lassen Sie mich daher nun beispielhaft im Abschluss die Diskussion auf eine Forschungsmethode konzentrieren, die es ermöglicht, Lebenswelten und Lebensräume empirisch zu entdecken und zu analysieren und gerade durch die Erweiterung der methodischen und analytischen Möglichkeiten durch die Datenwissenschaft eine tiefe Erweiterung ihrer analytischen Möglichkeiten erfahren hat: der Netzwerkanalyse. 


\subsection{Methodenkompetenzen am Beispiel der Netzwerkanalyse ${ }^{8}$}

Die Methodenwerkzeuge der empirisch-basierten Netzwerkanalyse haben sich in den letzten Jahrzehnten signifikant verändert, analog zu vielen anderen sozial-wissenschaftlich relevanten empirischen Forschungs- und Analysemethoden. Die gegenwärtige Bedeutung der Netzwerkanalyse in den Sozial- und Bildungswissenschaften ist vor allem auf ihre enge Verknüpfung mit dem theoretischen Konzept des Sozialkapitals zurückzuführen (Rürup, Röbken, Emmerich, \& Dunkake, 2015, S. 44). Netzwerkanalysen können Beziehungsstrukturen sichtbar machen, die jenseits der offiziellen und offensichtlichen Formen der Koordination von Akteuren und Handlungen in der Gesellschaft liegen, denen möglicherweise aber eine hohe Bedeutung für die Koordinationsleistung überhaupt zukommt (Rürup u. a., 2015, S. 65). Dieser Ansatz bietet als lang etablierte, in den letzten Jahren aber in ihrer Bedeutung durch ihre Anwendungspotenziale gestiegene Methode in Forschung und Praxis beste Möglichkeiten, hybride Lebensräume genauso gut darzustellen wie auch andere, ,klassische“ Lebenswelten Menschen aller Altersgruppen und Lebenssituationen. Stegbauer (2010) in seinem umfassenden Methodensammelband spricht sogar von einem ,neuen Paradigma in den Sozialwissenschaften“.

Die empirische Sozialforschung bietet heute eine große Vielfalt an qualitativen und quantitativen Methodenwerkzeuge an, um selbst komplexeste Lebensraumgestaltungsmuster empirisch zu erheben, zu analysieren und Theorieentwicklung oder -überprüfung auf eine empirisch sichere, datengestützte Grundlage zu stellen. Dies gilt mittlerweile auch dort, wo dies bislang äusserst schwierig war, also vor allem in den Bereichen, in denen hybride Welten — Lebensräume, die sich gleichermassen in analogen und digitalen Kontexten manifestieren - Forscherinnen und Forscher, aber eben gerade auch Praktikerinnen und Praktiker der Sozialen Arbeit enorme Hürden aufzeigten, diese Lebensräume zu entdecken, zu kartographieren und zu analysieren. Wie aber jede/r Soziale Arbeiter*in weiß, ist ein tiefes Verständnis der Lebenswelt von Klienten unerläßlich für eine wirksame und nachhaltige Intervention und Maßnahmengestaltung (vgl. Fuhse, 2010). Daher ist es eben nicht nur für die Forschung wichtig, mit diesen Methoden vertraut zu sein, sondern auch im sozialarbeiterischen Alltag.

Somit gilt: auch die mehr praxisfokussierte Ausbildung von Sozialarbeiter*innen bedingt mittlerweile ein methodisch breiter gefächertes Handwerkszeug, wenn etabliertes professionelles Handeln auch in zukünftigen, oftmals komplexeren Lebensräumen - bezogen auf deren Manifestation - Anwendung finden soll. Der weiter zunehmenden Druck auf die professionellen Akteure in der Sozialen Arbeit durch die Forderung nach immer mehr Evidenz-basierten Formen der Dokumentation und Begründung von Maßnahmen und deren Wirksamkeit tut hier sein übriges. Mit anderen Worten: ein sicherer Umgang mit empirischen Methoden wird immer stärker ein Faktor in Praxis und damit auch den Ausbildungsanforderungen an junge Sozialarbeiter*innen, unabhängig von ihrem jetzigen oder späteren Arbeitsfeld. Bodenhaftung und Feldkenntnis bedingen heute Grundlagen der Analyse, die noch vor zwanzig, dreißig Jahren hauptsächlich als Spielfeld der forschenden Kolleg*innen an den Universitäten und der Theorieentwicklung, im Alltag aber als wenig hilfreich angesehen wurden.

Die durch Netzwerkanalyse empirisch darstellbaren informellen Beziehungsstrukturen werden vor allem dann interessant, wenn es um die Frage geht, wie Handlungen gezielt koordiniert und Akteure in ihrem informellen Handeln beeinflusst werden können. Formal mögen Handlungsstrukturen klar abgebildet sein. Diese formalen Strukturen unterscheiden sich meist wenn auch in unterschiedlicher Spanne - von den darunterlegenden informellen Strukturen

\footnotetext{
${ }^{8}$ Im Kontext dieses Beitrags wird der Begriff „Netzwerkanalyse“ Synonym mit der Bedeutung der „Sozialen Netzwerkanalyse" verwandt, wenngleich methodisch hier durchaus Abgrenzungen festzustellen sind.
} 
die für den Erfolg bzw. Misserfolg von Handlungsprozessen oftmals wesentlich mehr Bedeutung haben als die formalen Strukturen.

Die Untersuchung sozialer und institutioneller Netzwerke stellt für die empirische Erfassung und das Verständnis von Sozialräumen gerade durch die Möglichkeit eines vielschichtigen, multidisziplinären oder ggf. auch interdisziplinären Ansatzes eine besonders geeignete Methode für die Soziale Arbeit dar. Über den Ansatz der Netzwerkanalyse kann der Sozialraum nicht nur aus sozialwissenschaftlicher Perspektive beleuchtet werden. Vielmehr lassen sich auch verhaltens- und erziehungswissenschaftlichen Perspektiven einbeziehen. Auf diese Weise lässt sich einerseits die räumliche Gestalt der sozialen Verbindungen, andererseits aber auch die inhaltlichen Formen der Konstruktion der Räume methodisch abbilden und analysieren. Somit lassen sich ganz wesentliche Aspekte für Handlungsoptionen identifizieren, die mit anderen Mitteln oftmals schwer erkennbar sind aber aus Sicht der Praktikerin und des Praktikers von ganz wesentlicher Bedeutung für eine erfolgreiche Arbeit sind.

Für Otto und Bauer (Bauer \& Otto, 2005; Otto \& Bauer, 2005) beispielsweise stellen soziale Netzwerke eine zentrale Perspektive für psychosoziale und gesundheitsbezogene Interventionen dar. Dies bedingt eine interdisziplinäre Sicht auf diese sozialen Netzwerke und somit die Einbeziehung benachbarter Teilgebiete wie Soziale Arbeit, Gesundheitsförderung, Pflege und Soziale Gerontologie. Die folgenden beiden Arbeiten sind dafür typische Beispiele:

Beispiel 1: Ryan, A. M. (2001). The peer group as a context for the development of young adolescent motivation and achievement. Child Development, 72(4), 1135-1150.

In dieser Arbeit untersucht Ryan (2001) explizit mit Methoden der Netzwerkanalyse die Entwicklung der Leistungsmotivation und -aspiration von Schülern in Abhängigkeit von der Motivation/Aspiration ihres Freundeskreises. Es zeigte sich, dass sich die individuelle Schulmotivation und die Leistungsaspirationen im Laufe eines Schuljahres an die Motivation der Peergroup angepasst; die individuelle Einstellung der Schüler, ob Schule an sich einen Nutzen hat oder nicht, blieb hingegen unbeeinflusst.

Beispiel 2: Flashman, J. (2012). Academic achievement and its impact on friend dynamics. Sociology of Education, 85(1), 61-80.

Flashman (2012) betrachtet in dieser quantitativen Studie den Einfluss schulischer Leistungen auf die Entwicklung und Dynamik von Freundschaftsbeziehungen. Sie stellt fest, dass Schüler mit guten bzw. defizitären Leistungen auch primär Freundschaftsnetzwerke zu leistungshomogenen Schülern aufbauen. In einer ,typischen“ amerikanischen Highschool ist die Wahrscheinlichkeit, dass leistungsstarke Schüler*innen Freundschaftsnetzwerke insbesondere mit anderen leistungsstarken Peers aufbauen hoch, unabhängig von anderen soziodemographischen Faktoren. Eine vergleichbare Dynamik zeigt sich auch für schulisch wenig erfolgreiche Schüler*innen, die ebenfalls eine höhere Wahrscheinlichkeit aufzeigen mit schulisch wenig erfolgreichen Peers Freundschaftsbeziehungen und -netzwerke aufzubauen und zu unterhalten. Ändert sich die schulische Leistung, ändern sich auch die Netzwerke und Freundschaftsbeziehungen der Jugendlichen. Dies gilt gleichermassen für Schüler*innen, deren Leistung sich verbessert oder verschlechtert.

Jede sozialarbeiterische Intervention muss aus dieser Erkenntnis heraus also zwingend beide Ebenen, die strukturelle Einbindung - hier: die Freundschaftsnetzwerke und Beziehungsgeflechte - und die individuelle Person gemeinsam in den Blick nehmen. Ein individualfokussierter Ansatz alleine der beispielsweise in der Vermittlung über die Rolle und Bedeutung schulischer Leistungen für spätere Lebensoptionen von Individuen, kann dem oder der 
Schüler*in nicht gerecht werden, vor allem, wenn eine Verbesserung der schulischen Kompetenz und Leistung innerhalb des Schulsystems dauerhaft sein soll.

Freeman (2004, S. 3) weisst auf vier Elemente der modernen Netzwerkanalyse als Forschungsprogramm bzw. -paradigma hin:

1. Social network analysis is motivated by a structural intuition based on ties linking social actors;

2. it is grounded in systematic empirical data;

3. it draws heavily on graphic imagery; and

4. it relies on the use of mathematical and/or computational models.

Aus dieser Aufzählung wird schnell deutlich, warum diese Methode für die Arbeit mit Lebenswelten von immenser Bedeutung ist, verbindet sie doch systematisch strukturelle Ebenen mit den Akteur*innen auf empirischer Datenbasis mit Mitteln der graphischen Darstellung, die einen vielfältigen Einsatz auch ohne große quantitative Komputationen ermöglicht. Gleichzeitig erlaubt die Erweiterung des Ansatzes auf Basis quantitativer Methoden auch wesentlich komplexere Analysen und eine Einbindung in lang etablierte Formen der Evidenzdarstellung, wie etwa der Korrelationsanalyse und dem Aufbau kausaler Zusammenhänge. Dies wiederum bedingt eine methodische Kompetenzerweiterung in der Lehrvermittlung um Studierenden die Möglichkeiten der auf computer-Modellen basierten Analyse auch im professionellen Alltagskontext zu erschliessen.

Ein wesentliches Element der Netzwerkanalyse als Methode ist somit ihre kombinatorische Grundanlage: zum einen beruht die Datenerfassung im Rahmen einer Netzwerkanalyse häufig auf der Anwendung qualitativer Forschungsmethoden, obwohl auch hier durchaus quantitative Abfragen eine breite Anwendung finden; zum anderen gibt es mittlerweile ein breites Arsenal quantitativer Analysemethoden dieser so erhobenen Daten, sowohl auf graphischer Basis, als auch in Verbindung mit traditionellen stochastischen Methoden. Die Netzwerkanalyse — vor allem in der für unser Einsatzgebiet zentralen Form, der Sozialen Netzwerkanalyse — bietet somit umfangreiche Möglichkeiten der Anwendung von Mixed-Methods Studiendesigns und somit Anwendung auch auf Felder, bei denen die Datenerhebung oftmals große Schwierigkeiten und Hürden aufweist.

Netzwerkanalysen sind nicht nur Instrumente der Sozialen Arbeit im Bereich der Forschung, sondern können auch im ganz normalen Alltag der professionell angewandten Sozialen Arbeit Anwendung finden. Als Analysewerkzeug erlaubt es Einblicke, die Transparenz für alle Beteiligten schafft und oft auch zur Kommunikation mit Klient*innen aber auch gegenüber Maßnahmenträgern gut geeignet ist, wesentliche Aspekte Evidenz-basiert darzustellen.

Da die Netzwerkanalyse auf Jahrzehnte der Forschung und Anwendung zurückblicken kann, hat sich eine Vielzahl an Anwendungsformen und methodischen Ausgestaltungen entwickelt. Insbesondere die Tradition der sozialen Netzwerkanalyse (Social Network Analysis) aus dem angelsächsischen Raum findet zunehmend Anwendung in einer Vielzahl verschiedner Sozialwissenschaften, aber auch vermehrt in verwandten Gebieten. Da soziale Netzwerkanalyse zwar eine gemeinsame Sprachlichkeit (,,vocabulary“) und Maße für die Beschreibung relationaler Daten und deren Analyse bietet aber keine Akzeptanz einer bestimmten Strukturtheorie voraussetzt (vgl. Scott, 2013, Kapitel 1, „Interpretation of Network Data“, Abs. 3), ist sie besonders attraktiv für die Anwendung in der Sozialen Arbeit. Hier wollen wir kurz auf zwei wesentliche Hauptlinien der Netzwerkanalyse fokussieren, die von besonderer Relevanz für die Soziale 
Arbeit sind: (1) Gesamtnetzwerkanalysen und (2) ego-zentrierte Netzwerkanalysen (für eine beispielhafte Anwendung, siehe Kulin, Frank, Fickermann, \& Schwippert, 2012).

\subsubsection{Gesamtnetzwerkanalysen}

Eine der Hauptlinien in der Netzwerkanalyse sind sog. Gesamtnetzwerkanalysen. Diese stellen eine Betrachtung der Beziehungen aus der Sicht aller beteiligten Akteure eines Systemzusammenhanges dar. Beispielhaft sind hier etwa zu nennen:

- Hilfenetzwerk zwischen allen Bewohner*innen eines Hauses

- Kommunikationsnetzwerk der Mitglieder einer Organisation

- Beziehungsnetzwerk innerhalb einer Schule

\subsubsection{Ego-zentrierte Netzwerkanalyse}

Die zweite große Linie von besonderer Bedeutung für die Soziale Arbeit sind sog. „,egozentrierte Netzwerkanalysen“". Ihr Schwerpunkt liegt auf der Beschreibung und Untersuchung der interpersonalen Umgebung eines Akteurs. Diese ego-zentrierten Netzwerke bilden „Beziehungen eines fokalen Akteurs (Ego) zu anderen Akteuren (Alteri) der direkten Netzwerkumgebung, sowie den Beziehungen zwischen diesen Akteuren (Alter-Alter-Beziehungen)“ ab (Herz, 2012, S. 134). ${ }^{9}$ Die so erstellten Netzwerkbilder erlauben eine visuelle, graphischbasierte Analyse der Form und Einbindung in die aus den Beziehungen entstandene Struktur sowohl des Egos, als auch der Alteri. Darüber hinaus gibt es aber seit längerer Zeit auch computer-basierte Methoden der quantitativen stochastischen Analyse dieser Beziehungen.

Innerhalb der ego-zentrierten Netzwerkanalyse sind zwei Formen der Erhebung und Analyse zu unterscheiden: (1) rein standardisierte Befragungsarten, in welchen anhand bestimmter Kriterien sowohl jene Beziehungen erfasst und untersucht werden, die eine bestimmte Referenzperson (Ego) mit anderen Netzwerkpersonen (Alteri) unterhält, als auch die Verbindungen der Alteri untereinander (Alter-Alter-Relationen) und (2) verschiedene Arten von Netzwerkkarten, die Interviewer und Interviewte gemeinsam im Befragungsablauf erstellen (Herz \& Gamper, 2012, S. 57).

\subsubsection{Beispiele}

Die folgenden beiden Beispiele sollen kurz einen Einblick geben in die praktische Ausgestaltung der sozialen Netzwerkmethode und deren Anwendung im professionellen Kontext.

Digitale Netzkarten Netzkartenerstellung mit VennMaker

Webatlas Sinus Nutzung des Webatlas Sinus zur Darstellung von Sozialräumen basierend auf physischer Lokalität und Sozialkapital

\footnotetext{
${ }^{9} \mathrm{Vgl}$. auch die Definition aus einem der einflussreichsten methodischen Werke in diesem Gebiet von Wasserman und Faust: ,,an ego-centered network consists of a focal actor, termed ego, as set of alters who have ties to ego, and measurements on the ties among these alters" (Wasserman \& Faust, 1994, S. 42).
} 
Lassen sie uns an dieser Stelle noch einmal kurz Revue passieren: der Sozialraumansatz findet in der Sozialen Arbeit in vielfältiger Form Anwendung, da er mehrebenenfähig, multi- und interdisziplinär eingesetzt werden kann. Zwei darin enthaltene Konzepte zeigen eine besonders enge Verknüpfung über eine gemeinsame Methode auf: das Sozialkapital und die Theorie der Lebenswelt lassen sich beide gemeinsam mit Hilfe der Netzwerkanalyse sowohl in der Forschung und Theorieentwicklung, als auch in der praktischen Sozialen Arbeit analytisch und für die Ermittlung von Interventionsansätzen verbindend einsetzen. Diese Vorteile werden vor allem hinsichtlich der zunehmenden, von Globalisierungs- und Digitalisierungsprozessen hervorgerufenen gesamtgesellschaftlichen Transformationen, relevant. Diesen Phänomenen und deren Konsequenzen für Forschung und praktische Soziale Arbeit widmen wir nun unsere Aufmerksamkeit um im Anschluss die daraus resultierenden Herausforderungen für Forschung und Ausbildung in der Sozialen Arbeit abschließend zu beleuchten.

\section{Herausforderungen für Lehre und Ausbildung: Digital- isierung und methodologische Kompetenzprofile}

Diese Arbeit hat einen Einblick gegeben in die methodischen Grundzüge und analytischen Möglichkeiten der sozialen Netzwerkanalyse im Rahmen einer Sozialraum-basierten Sozialen Arbeit. Globalisierungsprozesse, Migration, Digitalisierung und der sich stetig fortsetzende Trend zur Generierung von Daten und Erstellung von Dokumentationen im Rahmen der Evidenz-basierten professionellen Anforderungen an Soziale Arbeit machen es notwendig, Praktiker*innen in diesem Feld eine Methodenkompetenz zu vermitteln, die deren professionelles Handeln inmitten der stattfindenden transformatorischen Prozesse und den damit verbundenen Umwälzungen bezogen auf Kernelemente der Sozialen Arbeit im Bereich der Lebenswelt von Klient*innen auch in der Zukunft ermöglichen.

Abschliessend will ich einen Ausblick wagen, welche Konsequenzen all dies für Forschung, Ausbildung und Anwendung der Methoden der Sozialen Arbeit hat, bezogen auf die Erhebung von Daten, deren Analyse und deren Anwendung im Kontext einer sozialarbeiterischen Maßnahme bzw. Intervention.

Der Einfluß der Digitalisierung auf soziale Systeme kann gar nicht genug in der Ausbildung und in der Vorbereitung für den Alltag in der Sozialen Arbeit betont werden. Dies gilt sowohl für die Lebenswelt von Klient*innen, als auch für die administrativen Aufgaben und Anforderungen innerhalb der professionellen Sozialen Arbeitswelt. Die Ausbildung muss daher verschiedene Kompetenzprofile berücksichtigen, da sich diese Kompetenzen für die Erhebung und Analyse von Daten von denen in den administrativen Bereichen teilweise unterscheiden. Gemeinsam gilt für alle Bereiche jedoch eine verstärkte Betonung auf die Daten-Sprachfähigkeit (Data Literacy) in der Ausbildung der Sozialen Arbeit.

Gerade aus ihrer historischen Entwicklung heraus ist für die Soziale Arbeit ein partizipativer Ansatz - also eine aktive Begleitung der aktuellen gesamtgesellschaftlichen Debatte über die Ausgestaltung der Datafizierung in Verwaltung und anderen Institutionalisierungen von gesellschaftlichen Normierungen des Miteinanders verschiedener sozialer Akteure — hier dringend geboten. Wenn viele Teile der Gesellschaft immer stärker auf Grundlage von datengesteuerten Prozessen für sich und vor allem auch für andere Entscheidungen treffen und oftmals eigene Handlungsoptionen direkt oder indirekt beeinflussen oder beschränken, ist es umso 
wichtiger die Entstehung von Daten und den Werten, die in ihnen kodifiziert sind zu verstehen, zu begleiten und in deren Entwicklung selber aktiv teilzunehmen, denn:

when data appear to be so self-evident and big data seem to hold such promise of truth, it has never been more essential to remind ourselves what data are not seen, and what cannot be measured. (Baym, 2013, S. 7. Conclusion, Abs. 2)

Dies erfordert eine Veränderung der Ausbildungsprofile in der Sozialen Arbeit, die eine Sprachfähigkeit sowohl im digitalen als auch im Datenbereich bei den einzelnen Akteur*innen gewährleistet und somit erst eine aktive Begleitung in der öffentlichen Debatte und die Begleitung von Menschen und Entwicklung neuer Methoden der Unterstützung und Analyse zur Stärkung ihrer Handlungsmacht und Eigenständigkeit sicherstellt.

Im folgenden Teil will ich daher kurz abschliessend auf die verschiedenen Elemente eines Kompetenzprofils bezogen auf die in der Erstellung, Sammlung und Analyse von Daten entstehenden Fragestellungen und Herausforderungen von in diesen Daten enthaltenen Normen, ihrer Formung (Kodifizierung) und den für eine aktive Begleitung dieser Prozesse notwendigen Sprachfähigkeiten (Literacies) eingehen.

\subsection{Datenerstellung}

Ein wesentlicher, aber oft übersehener Faktor in einer immer stärker von Daten geprägten sozialen Realität ist die besondere Herausforderung, die Kontrolle über Datengeneration also deren Erhebung und Bereitstellung - behalten zu können. Dies bedingt den Erwerb der notwendigen Kompetenzen einerseits, anderseits aber auch ein Verständnis für die Bedeutung von einmal in analoger und besonders auch digitaler Datenform bereitgestellten Informationen für die damit verbundenen Personen, Gruppen und Institutionen. Diese Daten können erhebliche Konsequenzen für zukünftige Maßnahmen und Unterstützungsangebote haben und müssen daher mit größtmöglicher methodischer Sorgfalt und Professionalität erstellt und gesichert werden. Dies schliesst auch ganz wesentlich die zunehmenden ethischen und gesetzlich begründeten Herausforderungen an Datenerhebung und -sicherung ein.

\subsection{Sammlung von Daten}

Die Entwicklung hin zu Datafizierung vieler formaler und informeller gesellschaftlicher Ausgestaltungsprozesse erhöht ebenso die Bedeutung der Sammlung von Daten bezogen auf Zugang, Kontrolle der Richtigkeit, technischen und dokumentarischen Standards sowie rechtlichen Fragen des Umfangs der Sammlung und damit des in diesen Daten kodierten personenbezogenen Wissens. Hier ist es besonders wichtig bereits in der Ausbildung die Bedeutung dieser Fragen nicht nur theoretisch, sondern bezogen auf ihre praktische Auswirkungen in realen Kontexten zu vermitteln.

\subsection{Datenanalyse}

Module zur Erhebung und Analyse von Daten bilden bereits einen festen Bestandteil der Ausbildung der zukünftigen Akteure*innen der Sozialen Arbeit. Vor allem im qualitativen Bereich werden hier Formen der Datenerhebung durch Befragung und Beobachtung grundsätzlich 
vermittelt. Die quantitative Ausbildung beschränkt sich häufig auf fragebogengestützte Formen der Datenermittlung und grundsätzliche statistische Methoden. Hier ermöglichen neue Methoden und Werkzeuge der Analyse auch niederschwellige Zugänge bereits in der BachelorAusbildung, die bislang einen umfangreicheren Ausbildungsweg bedingten.

Um eine solche Ausbildung zu gewährleisten ist es jedoch notwendig, einen grundsätzlichen Konsens über verbindliche Standards in der Ausbildung idealerweise nicht als „Insellösung“ einzelner Ausbildungsstätten zu gestalten, sondern auf der Basis gemeinsamer und verbindlicher Grundstandards der hier vermittelten Kompetenzen in den Bereichen qualitativer und qualitativer Analysestandards. Dabei sollte hier unbedingt die Kooperation mit anderen Ausbildungsbereichen einerseits, als auch mit institutionellen Stakeholdern, also den Trägern und öffentlichen Verwaltungen stärker als schon bislang gesucht und ausgebaut werden. An verschiedenen Hochschulen, etwa in Göttingen, Mainz, oder der Leuphana Hochschule in Lüneburg gibt es bereits umgesetzte Modelle der hochschulweiten Ausbildung im Bereich der digitalen und Datensprachfähigkeit die fächerübergreifend Standards in diesen Bereichen vermittelt.

Hier ist die Profession gefordert sich aktiv auf gemeinsame Standards zu verständigen und diese nicht nur in der Ausbildung des akademischen Nachwuchses vertiefend zu bedingen und zu ermöglichen, sondern auch in der Fortbildung des aktuellen akademischen Personals diese Standards als aktive Methodenkompetenzen des Lehr- und Forschungskörpers sicherzustellen.

\section{Literaturverzeichnis}

Abidin, C., \& Brown, M. L. (Hrsg.). (2018). Microcelebrity around the globe: Approaches to cultures of internet fame. Bingley, UK: Emerald.

Althusser, L. (1971). Ideology and ideological state apparatuses. (Notes towards an investigation). In B. Brewster (Übers.), Lenin and philosophy and other essays (2. Aufl., S. 121-176). London: Monthly Review Press.

Auerswald, P. E. (2017). The code economy: A forty-thousand-year history. New York, NY: Oxford University Press.

Baack, S. (2015). Datafication and empowerment: How the open data movement rearticulates notions of democracy, participation, and journalism. Big Data \& Society, 2(2), 2053951715594634. https://doi.org/10.1177/2053951715594634

Baldwin, R. (2019). The globotics upheaval: Globalisation, robotics and the future of work. London: Hachette UK.

Barth, F. (Hrsg.). (1969). Ethnic groups and boundaries: The social organization of culture difference. Bergen: Little, Brown.

Bauer, P., \& Otto, U. (Hrsg.). (2005). Mit Netzwerken professionell zusammenarbeiten. Band 2: Institutionelle Netzwerke in Steuerungs- und Kooperationsperspektive (Bd. 2). Tübingen: dgvt-Verlag.

Baumgärtel, T. (2013, Juli 4). Das Ende der Utopie. Neue Züricher Zeitung. Abgerufen von https://www.nzz.ch/feuilleton/das-ende-der-utopie-1.18110281

Baym, N. K. (2013). Data not seen: The uses and shortcomings of social media metrics. First Monday, 18(10). https://doi.org/201310081411 
Beer, D. (2019). The data gaze: Capitalism, power and perception. Thousand Oaks, CA: SAGE.

Behrisch, L. (2006a). Vermessen, Zählen, Berechnen des Raums im 18. Jahrhundert. In L. Behrisch (Hrsg.), Vermessen, Zählen, Berechnen: die politische Ordnung des Raums im 18. Jahrhundert (S. 7-25). Frankfurt am Main: Campus Verlag.

Behrisch, L. (Hrsg.). (2006b). Vermessen, Zählen, Berechnen: die politische Ordnung des Raums im 18. Jahrhundert. Frankfurt am Main: Campus Verlag.

Berezan, O., Krishen, A. S., Agarwal, S., \& Kachroo, P. (2018). The pursuit of virtual happiness: Exploring the social media experience across generations. Journal of Business Research, 89, 455-461. https://doi.org/10.1016/j.jbusres.2017.11.038

Betts, L. R. (2016). Cyberbullying: Approaches, consequences and interventions. London: Palgrave Macmillan. https://doi.org/10.1057/978-1-137-50009-0

BMG. (2017). ePflege: Informations- und Kommunikationstechnologie für die Pflege. Berlin: Bundesministerium für Gesundheit (BMG).

Bourdieu, P. (1983). Ökonomisches Kapital, kulturelles Kapital, soziales Kapital. In R. Kreckel (Hrsg.), Soziale Ungleichheiten. Soziale Welt, Sonderband Nr. 2 (S. 183-198). Göttingen: Schwartz. https://doi.org/10.1007/978-3-531-18944-4_15

Bourdieu, P. (2000). Zur Soziologie der symbolischen Formen. (W. H. Fietkau, Übers.) (11. Aufl.). Frankfurt am Main: Suhrkamp. (Original work published 1974)

Bourdieu, P. (2017). Meditationen: zur Kritik der scholastischen Vernunft. (A. Russer, Übers.) (4. Aufl.). Frankfurt am Main: Suhrkamp. (Original work published 1997)

Böhnisch, L., \& Münchmeier, R. (1990). Pädagogik des Jugendraums: zur Begründung und Praxis einer sozialräumlichen Jugendpädagogik. Weinheim: Juventa.

Branch, J. (2011). Mapping the sovereign state: Technology, authority, and systemic change. International Organization, 65(1), 1-36. https://doi.org/10.1017/S0020818310000299

Brandtzaeg, P. B., \& Lüders, M. (2018). Time collapse in social media: Extending the context collapse. Social Media + Society, 4(1), 2056305118763349. https: //doi.org/10.1177/2056305118763349

Brotton, J. (1998). Trading territories: Mapping the early modern world. Ithaca: Cornell University Press.

Brubaker, R. (2006). Ethnicity without groups. Cambridge, MA: Harvard University Press.

Buckingham, D. (Hrsg.). (2008). Youth, identity, and digital media. Cambridge, MA: MIT Press.

Budde, W., \& Früchtel, F. (2006). Die Felder der Sozialraumorientierung - ein Überblick. In W. Budde, F. Früchtel, \& W. Hinte (Hrsg.), Sozialraumorientierung: Wege zu einer veränderten Praxis (S. 27-50). Wiesbaden: VS Verlag für Sozialwissenschaften. https://doi.org/10.1007/ 978-3-531-90393-4_2

Budde, W., Früchtel, F., \& Hinte, W. (Hrsg.). (2006). Sozialraumorientierung: Wege zu einer veränderten Praxis. Wiesbaden: VS Verlag für Sozialwissenschaften.

Burt, R. S. (2005). Brokerage and closure: An introduction to social capital. Oxford: Oxford University Press. 
Chen, S.-H. (Hrsg.). (2018). Big data in computational social science and humanities. Cham: Springer International Publishing. Abgerufen von //www.springer.com/de/book/ 9783319954646

Clark, J., \& Slyke, T. V. (2010). Beyond the echo chamber: Reshaping politics through networked progressive media. New York: The New Press.

Clarke, R., \& Greenleaf, G. (2017). Dataveillance regulation: A research framework (UNSW Law Research Paper No. 17-84). Sydney: University of New South Wales (UNSW) Law. https: //doi.org/10.2139/ssrn.3073492

Coleman, J. S. (1988). Social capital in the creation of human capital. American Journal of Sociology, 94, S95-S120. Abgerufen von http://www.jstor.org/stable/2780243

Coleman, J. S. (1994). Foundations of social theory. Cambridge, MA: Harvard University Press.

Cope, B., \& Kalantzis, M. (Hrsg.). (2000). Multiliteracies: Literacy learning and the design of social futures. London: Routledge.

Cortada, J. W. (2012). The digital flood: Diffusion of information technology across the United States, Europe, and Asia. New York: Oxford University Press.

Deinet, U. (2009a). Jugendeinrichtungen als Aneignungsräume. In U. Deinet (Hrsg.), Sozialräumliche Jugendarbeit: Grundlagen, Methoden und Praxiskonzepte (S. 115-132). Wiesbaden: VS Verlag für Sozialwissenschaften. https://doi.org/10.1007/978-3-531-91895-2_6

Deinet, U. (Hrsg.). (2009b). Sozialräumliche Jugendarbeit. Wiesbaden: VS Verlag für Sozialwissenschaften. https://doi.org/10.1007/978-3-531-91895-2

Deinet, U. (2011). Sozialraumorientierung zwischen Anspruch und Wirklichkeit. In H. Schmidt (Hrsg.), Empirie der Offenen Kinder- und Jugendarbeit (S. 159-177). Wiesbaden: VS. https: //doi.org/10.1007/978-3-531-92714-5_5

Deinet, U., \& Krisch, R. (2002). Der sozialräumliche Blick der Jugendarbeit: Methoden und Bausteine zur Konzeptentwicklung und Qualifizierung. Wiesbaden: VS Verlag für Sozialwissenschaften.

Deinet, U., Szlapka, M., \& Witte, W. (Hrsg.). (2008). Qualität durch Dialog: Bausteine kommunaler Qualitäts- und Wirksamkeitsdialoge. Wiesbaden: VS Verlag für Sozialwissenschaften.

De Mauro, A., Greco, M., \& Grimaldi, M. (2015). What Is Big Data? A Consensual Definition and a Review of Key Research Topics. AIP Conference Proceedings, 1644(1), 97-104. https: //doi.org/10.1063/1.4907823

Dey, B. L., Balmer, J. M. T., Pandit, A., \& Saren, M. (2018). Selfie appropriation by young British South Asian adults: Reifying, endorsing and reinforcing dual cultural identity in social media. Information Technology \& People, 31(2), 482-506. https://doi.org/10.1108/ITP-08-2016-0178

Dijck, J. van. (2014). Datafication, dataism and dataveillance: Big Data between scientific paradigm and ideology. Surveillance \& Society, 12(2), 197-208. https://doi.org/10.24908/ss. v12i2.4776

Dutton, W. H., \& Shepherd, A. (2006). Trust in the Internet as an experience technology. Information, Communication \& Society, 9(4), 433-451. https://doi.org/10.1080/ 13691180600858606 
Earle, T. C., \& Cvetkovich, G. (1995). Social trust: Toward a cosmopolitan society. Westport, CT: Praeger.

Elder-Vass, D. (2016). Profit and gift in the digital economy. Cambridge: Cambridge University Press.

Emirbayer, M., \& Mische, A. (1998). What Is agency? American Journal of Sociology, 103(4), 962-1023. https://doi.org/10.1086/231294

Flashman, J. (2012). Academic achievement and its impact on friend dynamics. Sociology of Education, 85(1), 61-80. https://doi.org/10.1177/0038040711417014

Floridi, L. (2014). The fourth revolution: How the infosphere is reshaping human reality. New York: Oxford University Press.

Ford, M. (2015). Rise of the robots: Technology and the threat of a jobless future. New York: Basic Books.

Foster, I. (Hrsg.). (2017). Big data and social science: A practical guide to methods and tools. Boca Raton, FL: CRC Press Taylor \& Francis Group.

Fourcade, M., \& Healy, K. (2013). Classification Situations: Life-Chances in the Neoliberal Era. Accounting, Organizations and Society, 38(8), 559-572. https://doi.org/10.1016/j.aos. 2013.11.002

Freeman, L. C. (2004). The development of social network analysis: A study in the sociology of science. Vancouver, BC: Empirical Press.

Früchtel, F., Cyprian, G., \& Budde, W. (2013). Sozialer Raum und soziale Arbeit. Textbook: theoretische Grundlagen (3., überarb. Aufl). Wiesbaden: Springer VS.

Fuchs, C. (2014). Digital labour and Karl Marx. New York, NY: Routledge.

Fuchs, C., \& Mosco, V. (Hrsg.). (2016). Marx in the age of digital capitalism. Leiden: Brill.

Fuhse, J. (2010). Netzwerke und soziale Ungleichheit. In C. Stegbauer (Hrsg.), Netzwerkanalyse und Netzwerktheorie: Ein neues Paradigma in den Sozialwissenschaften (S. 79-90). Wiesbaden: VS Verlag für Sozialwissenschaften. https://doi.org/10.1007/978-3-531-92029-0_5

Galliers, R. D., Newell, S., Shanks, G., \& Topi, H. (2017). Datification and its human, organizational and societal effects: The strategic opportunities and challenges of algorithmic decisionmaking. The Journal of Strategic Information Systems, 26(3), 185-190. https://doi.org/10.1016/ j.jsis.2017.08.002

Gambetta, D. (Hrsg.). (1988). Trust: Making and breaking cooperative relations. New York: Blackwell.

Giddens, A. (1979). Central problems in social theory: Action, structure and contradiction in social analysis. Basingstoke: Macmillan.

Giddens, A. (1990). The consequences of modernity. Cambridge, UK: Polity.

Gitelman, L. (Hrsg.). (2013). "Raw data" is an oxymoron. Cambridge, MA: The MIT Press.

Goodfellow, R. (2011). Literacy, literacies, and the digital in higher education. Teaching in Higher Education, 16, 131-144. https://doi.org/10.1080/13562517.2011.544125

Gorz, A. (2010). The immaterial: Knowledge, value and capital. London: Seagull Books.

Granovetter, M. (1985). Economic action and social structure: The problem of embeddedness. American Journal of Sociology, 91(3), 481-510. https://doi.org/10.1086/228311 
Graßhoff, G. (Hrsg.). (2013). Adressaten, Nutzer, Agency: akteursbezogene Forschungsperspektiven in der sozialen Arbeit. Wiesbaden: Springer VS.

Guerrero-Pico, M., Masanet, M.-J., \& Scolari, C. A. (2018). Toward a typology of young produsers: Teenagers' transmedia skills, media production, and narrative and aesthetic appreciation. New Media \& Society, Advance Online Publication, 1-18. https://doi.org/10.1177/ 1461444818796470

Günther, W. A., Rezazade Mehrizi, M. H., Huysman, M., \& Feldberg, F. (2017). Debating big data: A literature review on realizing value from big data. The Journal of Strategic Information Systems, 26(3), 191-209. https://doi.org/10.1016/j.jsis.2017.07.003

Habermas, J. (1988). Theorie des kommunikativen Handelns - Band II: Zur Kritik der funktionalistischen Vernunft (10. Aufl, Bd. 2). Frankfurt/Main: Suhrkamp. (Original work published 1981)

Hammel, E. (Hrsg.). (2015). Der Aufbruch in die Infosphäre: Essays aus den Anfängen der Netzwerk-Gesellschaft. Framersheim: Brighton.

Hattler, J., \& Thomas, H. (2016). Medienumbruch und Öffentlichkeit. Münster: LIT Verlag Münster.

Häußling, R. (2010). Zur Verankerung der Netzwerkforschung in einem methodologischen Relationalismus. In C. Stegbauer (Hrsg.), Netzwerkanalyse und Netzwerktheorie: Ein neues Paradigma in den Sozialwissenschaften (S. 65-78). Wiesbaden: VS Verlag für Sozialwissenschaften. https://doi.org/10.1007/978-3-531-92029-0_4

Heidrich, J., Bauer, F., Iese, F., \& Krupka, D. (2018). Future Skills: Ansätze zur Vermittlung von Data Literacy in der Hochschulbildung (Arbeitspapier No. 37) (S. 114). Berlin: Hochschulforum Digitalisierung. https://doi.org/10.5281/zenodo.1413119

Herz, A. (2012). Erhebung und Analyse ego-zentrierter Netzwerke. In S. Kulin, K. Frank, D. Fickermann, \& K. Schwippert (Hrsg.), Soziale Netzwerkanalyse: Theorie, Methoden, Praxis (S. 133-152). Münster: Waxmann.

Herz, A., \& Gamper, M. (2012). Möglichkeiten und Grenzen der Erhebung ego-zentrierter Netzwerke im Online-Fragebogen und über digitale Netzwerkkarten. In M. Gamper, L. Reschke, \& M. Schönhuth (Hrsg.), Knoten und Kanten 2.0: soziale Netzwerkanalyse in Medienforschung und Kulturanthropologie. Bielefeld: Transcript.

Herzog, L. (2019). Die Rettung der Arbeit: ein politischer Aufruf. Berlin: Hanser Berlin.

Hintz, A., Dencik, L., \& Wahl-Jorgensen, K. (2018). Digital citizenship in a datafied society. Medford, MA: Polity Press.

Holtzhausen, D. (2016). Datafication: Threat or opportunity for communication in the public sphere? Journal of Communication Management, 20(1), 21-36. https://doi.org/10.1108/ JCOM-12-2014-0082

Howard, P. N. (2010). The digital origins of dictatorship and democracy: Information technology and political islam. Oxford University Press.

Ifrah, G. (2000). The Universal History of Numbers: From Prehistory to the Invention of the Computer. New York: Wiley.

Jenkins, H. (2009). Confronting the challenges of participatory culture: Media education for the 21 st century. Cambridge, MA: The MIT Press. 
Kelleher, J. D., \& Tierney, B. (2018). Data Science. Cambridge, MA: MIT Press.

Kitchin, R. (2014). The data revolution: Big data, open data, data infrastructures \& their consequences. Los Angeles: SAGE.

Kocka, J. (2014). Geschichte des Kapitalismus (Kindle Version). München: Beck. Abgerufen von https://www.amazon.de

Kollock, P. (1999). The production of trust in online markets. In E. J. Lawler, M. W. Macy, S. R. Thye, \& H. A. Walker (Hrsg.), Advances in group processes (Bd. 16, S. 99-123). Greenwich, CT: JAI.

Kramp, L., Novy, L., Ballwieser, D., \& Wenzlaff, K. (Hrsg.). (2013). Journalismus in der digitalen Moderne: Einsichten - Ansichten - Aussichten. Wiesbaden: Springer-Verlag.

Krisch, R. (2009). Sozialräumliche Methodik der Jugendarbeit: aktivierende Zugänge und praxisleitende Verfahren. Weinheim: Juventa.

Kulin, S., Frank, K., Fickermann, D., \& Schwippert, K. (Hrsg.). (2012). Soziale Netzwerkanalyse: Theorie, Methoden, Praxis. Münster: Waxmann.

Lane, J., Stodden, V., Bender, S., \& Nissenbaum, H. (Hrsg.). (2014). Privacy, big data, and the public good. Cambridge: Cambridge University Press.

Lange, P. G. (2014). Kids on YouTube: Technical identities and digital literacies. Walnut Creek,CA: Left Coast Press.

Lankshear, C., \& Knobel, M. (2006). New literacies: Changing knowledge and classroom learning (2. Aufl.). Buckingham: Open University Press.

Lankshear, C., \& Knobel, M. (Hrsg.). (2008). Digital literacies: Concepts, policies and practices (Bd. 30). New York: Peter Lang.

Lankshear, C., \& Knobel, M. (2011). New literacies: Changing knowledge and classroom learning (3. Aufl.). London: Open University Press.

Lee, M. K. O., \& Turban, E. (2001). A trust model for consumer internet shopping. International Journal of Electronic Commerce, 6(1), 75-91. https://doi.org/10.1080/10864415.2001. 11044227

Lewicki, R. J., McAllister, D. J., \& Bies, R. J. (1998). Trust and distrust: New relationships and realities. Academy of Management Review, 23(3), 438-458. https://doi.org/10.2307/259288

Li, Q., Cross, D., \& Smith, P. K. (Hrsg.). (2012). Cyberbullying in the global playground: Research from international perspectives. Chichester, UK: Wiley-Blackwell.

Lin, N., Cook, K. S., \& Burt, R. S. (Hrsg.). (2001). Social capital: Theory and research. New York: Routledge.

Luhmann, N. (2014). Vertrauen: Ein Mechanismus der Reduktion sozialer Komplexität (5. Aufl.). Konstanz: UTB. (Original work published 1968)

Lunn, R. J., \& Suman, M. W. (2008). Experience and trust in online shopping. In B. Wellman \& C. A. Haythornthwaite (Hrsg.), The Internet in Everyday Life (S. 549-577). Malden, MA: Blackwell. https://doi.org/10.1002/9780470774298.ch19

Madhavan, P., \& Wiegmann, D. A. (2007). Similarities and differences between human-human and human-automation trust: An integrative review. Theoretical Issues in Ergonomics Science, 8(4), 277-301. https://doi.org/10.1080/14639220500337708 
Marbach, J. H. (2010). Netzwerk und Sozialkapital. Dynamische Zusammenhänge im Licht von Paneldaten der Umfrageforschung. In C. Stegbauer (Hrsg.), Netzwerkanalyse und Netzwerktheorie: Ein neues Paradigma in den Sozialwissenschaften (S. 347-358). Wiesbaden: VS Verlag für Sozialwissenschaften. https://doi.org/10.1007/978-3-531-92029-0_27

Marjanovic, O., \& Cecez-Kecmanovic, D. (2017). Exploring the tension between transparency and datification effects of open government IS through the lens of Complex Adaptive Systems. The Journal of Strategic Information Systems, 26(3), 210-232. https://doi.org/10.1016/j.jsis. 2017.07.001

Markus, M. L. (2017). Datification, organizational strategy, and IS research: What's the score? The Journal of Strategic Information Systems, 26(3), 233-241. https://doi.org/10.1016/j.jsis. 2017.08.003

Martin, A. (2008). Digital literacy and the "digital society". In C. Lankshear \& M. Knobel (Hrsg.), Digital literacies: Concepts, policies and practices (Bd. 30, S. 151-176). New York: Peter Lang.

Mason, P. (2015). Postcapitalism: A guide to our future. London: Allen Lane.

Mayer-Schönberger, V. (2011). Delete: The virtue of forgetting in the digital age. Princeton, NJ: Princeton University Press.

Mayer-Schönberger, V., \& Ramge, T. (2018). Reinventing capitalism in the age of big data. New York: Basic Books. Abgerufen von https://www.overdrive.com/search?q= 99AB332F-9A1D-4843-A697-AD51CD768D5A

McInroy, L. B., \& Craig, S. L. (2018). Online fandom, identity milestones, and selfidentification of sexual/gender minority youth. Journal of LGBT Youth, 15(3), 179-196. https://doi.org/10.1080/19361653.2018.1459220

Menninger, K. (1958). Zahlwort und Ziffer: eine Kulturgeschichte der Zahl (2. Aufl.). Göttingen: Vandenhoeck \& Ruprecht.

Muellerleile, C., \& Robertson, S. (2018). Digital Weberianism: Bureaucracy, information, and the techno-rationality of neoliberal capitalism. Indiana Journal of Global Legal Studies, 25(1), Article 9. Abgerufen von https://www.repository.law.indiana.edu/ijgls/vol25/iss 1/9

Müller, D., Ligensa, A., \& Gendolla, P. (Hrsg.). (2009). Leitmedien: Konzepte - Relevanz Geschichte (Bd. 1). Bielefeld: transcript Verlag.

OECD (Hrsg.). (2000). Literacy in the information age: Final report of the International Adult Literacy Survey. Paris: Organisation for Economic Co-operation and Development (OECD).

OECD. (2019a). Data in the digital age (Going Digital Policy Note). Paris: Organisation for Economic Co-operation and Development (OECD). Abgerufen von https: //www.oecd.org/going-digital/data-in-the-digital-age.pdf

OECD. (2019b). How's life in the digital age?: Opportunities and risks of the digital transformation for people's well-being. Paris: Organisation for Economic Co-operation and Development (OECD). https://doi.org/10.1787/9789264311800-en

Otto, H.-U., \& Ziegler, H. (Hrsg.). (2010). Capabilities - Handlungsbefähigung und Verwirklichungschancen in der Erziehungswissenschaft (2. Aufl.). Wiesbaden: VS.

Otto, U., \& Bauer, P. (Hrsg.). (2005). Mit Netzwerken professionell zusammenarbeiten. Band 1: Soziale Netzwerke in Lebenslauf- und Lebenslagenperspektive (Bd. 1). Tübingen: dgvt-Verlag. 
Paus, E., \& Ford, M. (Hrsg.). (2018). Confronting dystopia: The new technological revolution and the future of work. Ithaca, NY: ILR Press.

Potter, J., \& McDougall, J. (2017). Digital media, culture and education: Theorising third space literacies. London: Palgrave Macmillan.

Raby, R. (2007). Across a great gulf? Conducting research with adolescents. In A. L. Best (Hrsg.), Representing youth: Methodological issues in critical youth studies (S. 39-59). New York: New York University Press.

Raley, R. (2013). Dataveillance and countervailance. In L. Gitelman (Hrsg.), "Raw data" is an oxymoron (S. 121-145). Cambridge, MA: The MIT Press.

Ridsdale, C., Rothwell, J., Smit, M., Ali-Hassan, H., Bliemel, M., Irvine, D., ... Wuetherick, B. (2015). Strategies and best practices for data literacy education: Knowledge synthesis report (Report). https://doi.org/10.13140/RG.2.1.1922.5044

Rudder, C. (2014). Dataclysm: Who we are when we think no one's looking. New York, NY: Crown.

Rullani, E. (2011). Ökonomie des Wissens: Kreativität und Wertbildung im Netzwerkkapitalismus. (K. Neundlinger, Übers.). Wien: Turia + Kant.

Rürup, M., Röbken, H., Emmerich, M., \& Dunkake, I. (2015). Netzwerke im Bildungswesen: eine Einführung in ihre Analyse und Gestaltung. Wiesbaden: Springer VS.

Ryan, A. M. (2001). The peer group as a context for the development of young adolescent motivation and achievement. Child Development, 72(4), 1135-1150. https://doi.org/10.1111/ $1467-8624.00338$

Schiller, D. (1999). Digital capitalism: Networking the global market system. Cambridge, MA: MIT Press.

Schneider, J. (2018). „Ausländer“ (Foreigners), Migrants, or New Germans? Identity-Building Processes and School Socialization Among Adolescents From Immigrant Backgrounds in Germany. New Directions for Child and Adolescent Development, 2018(160), 59-73. https://doi. org/10.1002/cad.20241

Scott, J. (2013). Social network analysis (3rd ed.). Los Angeles: SAGE. Abgerufen von http: //www.amazon.de

Sennett, R. (2007). The culture of the new capitalism. New Haven, CT: Yale University Press.

Sevignani, S. (2017). Surveillance, classification, and social inequality in informational capitalism: The relevance of exploitation in the context of markets in information. Historical Social Research / Historische Sozialforschung, 42(1 (159)), 77-102. https://doi.org/10.2307/ 44176025

Skiena, S. S. (2017). The data science design manual. Cham: Springer International. https: //doi.org/10.1007/978-3-319-55444-0

Skrobanek, J. (2016). Gruppe. In A. Scherr (Hrsg.), Soziologische Basics: Eine Einführung für pädagogische und soziale Berufe (S. 111-120). Wiesbaden: Springer Fachmedien Wiesbaden. https://doi.org/10.1007/978-3-658-11928-7_11

Skutta, S., \& Steinke, J. (Hrsg.). (2019). Digitalisierung und Teilhabe: Mitmachen, mitdenken, mitgestalten! Sonderband 2018 der Zeitschriften Blätter der Wohlfahrtspflege und Sozialwirtschaft. Wiesbaden: Nomos. 
Slonje, R., \& Smith, P. K. (2008). Cyberbullying: Another main type of bullying? Scandinavian Journal of Psychology, 49(2), 147-154. https://doi.org/10.1111/j.1467-9450.2007.00611.x

Smith, P. K., Mahdavi, J., Carvalho, M., Fisher, S., Russell, S., \& Tippett, N. (2008). Cyberbullying: Its nature and impact in secondary school pupils. Journal of Child Psychology and Psychiatry, 49(4), 376-385. https://doi.org/10.1111/j.1469-7610.2007.01846.x

Smith, W. D. (1984). The function of commercial centers in the modernization of european capitalism: Amsterdam as an information exchange in the seventeenth century. The Journal of Economic History, 44(4), 985-1005. https://doi.org/en

Spatscheck, C., Arnegger, M., Kraus, S., \& Mattner, A. (2008). Soziale Arbeit und Ökonomisierung: Analysen und Handlungsstrategien. Berlin: Schibri.

Srnicek, N. (2017). Platform capitalism. Cambridge, UK: Polity.

Staab, P. (2016). Falsche Versprechen: Wachstum im digitalen Kapitalismus. Hamburg: Hamburger Edition.

Stegbauer, C. (Hrsg.). (2010). Netzwerkanalyse und Netzwerktheorie: Ein neues Paradigma in den Sozialwissenschaften (2. Aufl.). Wiesbaden: VS Verlag für Sozialwissenschaften. https: //doi.org/10.1007/978-3-531-92029-0

Støle, H. (2018). Why digital natives need books: The myth of the digital native. First Monday, 23(10). https://doi.org/10.5210/fm.v23i10.9422

Sztompka, P. (1999). Trust: A sociological theory. Cambridge: Cambridge University Press.

Taylor, L., \& Broeders, D. (2015). In the name of Development: Power, profit and the datafication of the global South. Geoforum, 64, 229-237. https://doi.org/10.1016/j.geoforum.2015.07. 002

Thrift, N. J. (2005). Knowing capitalism. London: SAGE.

Wajcman, J. (2015). Pressed for time: The acceleration of life in digital capitalism. Chicago: University of Chicago Press.

Wasserman, S., \& Faust, K. (1994). Social network analysis: Methods and applications. Cambridge: Cambridge University Press.

Wellman, B., \& Haythornthwaite, C. A. (Hrsg.). (2002). The Internet in everyday life. Malden, MA: Blackwell.

Westlund, H. (2006). Social capital in the knowledge economy: Theory and empirics. Berlin: Springer Berlin Heidelberg.

Wright, A. (2007). Glut: Mastering information through the ages. Washington, DC: Joseph Henry Press.

Wu, T. (2017). The attention merchants: The epic scramble to get inside our heads. New York: Vintage Books, a division of Penguin Random House LLC.

Zhang, L. (2018). The contradictions of ,women's work“ in digital capitalism: A „non-Western“/Chinese perspective. Feminist Media Studies, 18(1), 147-151. https: //doi.org/10.1080/14680777.2018.1407121

Zuboff, S. (2018). The age of surveillance capitalism: The fight for a human future at the new frontier of power. New York: PublicAffairs. 